\title{
Improved Time Domain Substructural Damage Identification Method on Large-Span Spatial Structure
}

\author{
Taoyuan Yang, ${ }^{1}$ Kun Liu, ${ }^{2,3}$ and Guibo Nie $\mathbb{D}^{1}$ \\ ${ }^{1}$ Key Laboratory of Earthquake Engineering and Engineering Vibration, Institute of Engineering Mechanics, \\ China Earthquake Administration, Harbin 150000, China \\ ${ }^{2}$ Key Lab of Structures Dynamics Behavior and Control of the Ministry of Education, Harbin Institute of Technology, \\ Harbin 150090, China \\ ${ }^{3}$ Key Lab of Smart Prevention and Mitigation of Civil Engineering Disasters of the Ministry of Industry and Information \\ Technology, Harbin Institute of Technology, Harbin 150090, China
}

Correspondence should be addressed to Guibo Nie; nieguibo0323@163.com

Received 12 August 2021; Revised 1 October 2021; Accepted 6 October 2021; Published 23 October 2021

Academic Editor: Chengwei Fei

Copyright (C) 2021 Taoyuan Yang et al. This is an open access article distributed under the Creative Commons Attribution License, which permits unrestricted use, distribution, and reproduction in any medium, provided the original work is properly cited.

The large-span spatial structure is a complex structural type with large number of elements, which makes health monitoring difficult. A time domain global substructural identification method was proposed in this paper to identify the local damage for the large-span spatial structure. The proposed method was an improvement method based on the time domain substructural identification method, which can identify the damage with a reduced structural model, and explicit force identification method, which can give convergent force identification result with incomplete response measurements, and it can assess one or more target substructures without knowing the conditions of the other parts of the structure. The application of the new method to large-span spatial structure was presented, and then an improved global method was proposed to further reduce the computation time and promote it in practice. Two orthogonal spatial square pyramid grid structure models are constructed to validate the time domain global substructural method and the improved global method. The results show that the time domain global substructural damage identification method identifies the small local damage in multiple members with satisfactory accuracy and the improved global method effectively shortens identification time.

\section{Introduction}

A great quantity of large-span spatial structures, such as stadiums, train stations, airport terminals, and exhibition centers, have been constructed around the world in the past several decades. The large-span spatial structure usually has important functions to undertake activities or transfer passengers, thereby always gathering large numbers of people in it. Once failure or even collapse occurs, it will cause terrible casualties, huge property loss [1], and extremely bad social impact, which makes the structural health monitoring of large-span spatial structure a crucial issue. The small local damage may lead to disastrous damage under strong earthquake, so the small local damage detection becomes a key issue for the structural health monitoring of the largespan spatial structure. The damage detection method based on vibration information [2-5] has been proposed and developed in the past three decades, and it has been applied in various structures (e.g., bridges [6], frame structures, and plane trusses $[7,8])$.

Several structural health monitoring methods based on vibration information have been proposed to estimate the local damage of space truss structure in recent years. The square ratio of frequency variation and variation rate of axial strain were adopted to identify local damage of space truss structure $[9,10]$, and the numerical simulation results show that the single damage location can be detected accurately while the damage quantification needs to be further discussed. Song et al. [11] expanded incomplete mode shape through a dynamic model expansion technique, identified the possible damage members by model strain energy, and then used Least Squares Support Vector 
Machine to detect the location and extent of the local damage of a space truss. However, the measurement noise level is a challenge. Another method based on residual modal force and modal strain energy was proposed to detect the local damage of space truss [12], and a space truss was numerically simulated to verify the proposed method. The damage location and quantification can be detected accurately, but the sensitive mode shape is needed in the simulation cases.

The neural networks have been adopted in structural health monitoring widely since last decades. Wu and Zhang [13] presented the damage identification method of grid structures based on BP neural networks, and it has been verified with numerical model of space truss and experimental model of a double-layer cylindrical reticulated shell structure. The erroneous judgment is always unavoidable for some damage locations, and this method cannot give good identification results with missed damaged members. To solve the data explosion problem, Liu et al. [14] introduced the method of substructure, while using probabilistic neural networks (PNN) to improve identification accuracy. The numerical simulation results show that this method is effective, but there are still erroneous judgments. If the damaged members are located in different substructures, the condition assessment will become more complicated, and excessive substructure partitioning will produce some new problems in global detection. He and Yan [15] adopted the method combining wavelet packet with support vector machine to identify the damage of single-layer lattice shell, but the comprehensive correct rate of damage identification was $80 \%$.

Data fusion technique has also been adopted for the damage detection of large-span spatial structure to improve the accuracy of the damage identification. The information of acceleration measurements and strain measurements is used to identify the damage of reticulated shell based on Dempster-Shafer evidence theory $[16,17]$. Teng and Yao [18] added wavelet packet analysis on the basis of information fusion. Although both methods improve the accuracy of damage identification, for avoiding data explosion, global structure still needs to be divided into too many parts. Moreover, the damaged members are all located in the same substructure; the scenarios with damaged members located in different substructures are not considered. Therefore, these methods can be used to assess a small amount of concentrated damage.

A probabilistic substructure identification and health monitoring methodology was proposed [19], which does not require any interface measurements or excitation measurements. This method can be applied widely, because only the stochastic model of the input is required. Then, the boundary force in the substructure is modulated as filtered white noise [20], which can be viewed as a continuity condition. This proposed method does not require stationarity of the response. An identifiability-enhanced Bayesian frequency-domain substructure identification approach is proposed without the requirement of input or boundary force measurements [21], in which extra constraints are imposed to enhance the identifiability of the inverse problem. Substructural identification approaches provide effective methodologies for the identification of large-span spatial structures, because they offer the flexibility to isolate some critical substructures for identification. However, for large-span spatial structure, the common substructural damage identification methods based on vibration information still suffer difficulties because of the characteristics of large-span spatial structure including complex structural composition, intensive frequency distribution, and numerous dynamic degrees of freedom.

The response sensitivity-based method was derived by Jahn [22] in 1940s; usually, its application involves model updating iteration and optimization algorithm [23-25] to enhance the identifiability. Liu et al. [26] proposed a substructural condition assessment method based on response sensitivity, and the accuracy and effectiveness are then validated with simulation studies of a plane truss when damage only exists in one of the substructures. This method, with comparatively lesser substructure partitioning, may be suitable for large complex structures. Since this method shows potential for damage identification of large-span spatial structure, in this paper, the global damage identification method based on this substructural method is presented to detect the location and extent of local damage in different substructures with uncompleted acceleration measurements. An improved global substructural damage identification method is further proposed to reduce calculation duration. The global substructural damage identification method and the improved global substructural damage identification method are numerically verified with simulation studies of two double-layer lattice space structures.

\section{The Global Substructural Damage Identification Method}

The target substructure can be assessed based on the time domain response sensitivity matrix of the substructural system as is described in Appendix B, and the detailed process has been written in previous paper [26]. However, this original substructural method is effective only when the condition of the rest substructure is known. The interface force depends on the local damage extent of the target substructure and the rest substructure, so the interface force sensitivity is related to the local damaged parameters of both substructures. When the local damaged parameter of the rest substructure is unknown, the substructural response sensitivity considering the interface force effect cannot be obtained. To overcome this problem, this paper will propose a global substructural method based on the interrelation between each substructural dynamic equation.

If the whole structure is divided into $n$ substructures, similar to (B.6), the motion equation of $i$ th substructure among the $n$ substructures can be written as

$$
M_{i} \ddot{x}_{i}+C_{i} \dot{x}_{i}+K_{i} x_{i}=L_{i} P_{i}
$$

where the subscripts $i$ denote the number of substructures; obviously $1 \leq i \leq n$. The interface force $P_{i}$ is related to all the 
substructural stiffness matrices from $K_{1}$ to $K_{n}$. Due to the interconnection between substructural motion equations, the global substructural dynamic equation set can be written as

$$
\left\{\begin{array}{c}
M_{1} \ddot{x}_{1}+C_{1} \dot{x}_{1}+K_{1} x_{1}=L_{1} P_{1} \\
\vdots \\
M_{i} \ddot{x}_{i}+C_{i} \dot{x}_{i}+K_{i} x_{i}=L_{i} P_{i} \\
\vdots \\
M_{n} \ddot{x}_{n}+C_{n} \dot{x}_{n}+K_{n} x_{n}=L_{n} P_{n} .
\end{array}\right.
$$

In (2) the DOFs of the interface are repeated once compared with the equation of motion of the full structure.

2.1. The Global Substructural Damage Identification Algorithm. Assume that the local damage extent of the $j$ th substructure is damage index vector $\alpha_{j}$, which includes the local damaged parameter of every element in the $j$ th substructure. A change in the global substructural stiffness matrix can be described as

$$
\Delta K=\sum_{j} \alpha_{j} K_{j} .
$$

Performing differentiation of both sides of (1), the motion equation of $i$ th substructure, with respect to the damage index vector $\alpha_{j}$, we have

$$
M_{i} \frac{\partial \ddot{x}_{i}}{\partial \alpha_{j}}+C_{i} \frac{\partial \dot{x}_{i}}{\partial \alpha_{j}}+K_{i} \frac{\partial x_{i}}{\partial \alpha_{j}}=-\frac{\partial K_{i}}{\partial \alpha_{j}} x_{i}-a_{1} \frac{\partial K_{i}}{\partial \alpha_{j}} \dot{x}_{i}+L_{i} \frac{\partial P_{i}}{\partial \alpha_{j}},
$$

where $\partial \ddot{x}_{i} / \partial \alpha_{j}, \partial \dot{x}_{i} / \partial \alpha_{j}$, and $\partial x_{i} / \partial \alpha_{j}$ are the substructural response sensitivity matrices which can be obtained by solving (4). The substructural response sensitivity algorithm is presented in (B.9)-(B.16).

Let the $i$ th substructural response sensitivity matrix with respect to the $j$ th substructural damage index vector be represented as $S_{i, j}$. All the substructural sensitivity matrices can be calculated, and then the global substructural sensitivity matrix is assembled as

$$
S=\left[\begin{array}{ccccc}
S_{1,1} & \cdots & S_{1, j} & \cdots & S_{1, n} \\
\vdots & \ddots & \vdots & \ddots & \vdots \\
S_{i, 1} & \cdots & S_{i, j} & \cdots & S_{i, n} \\
\vdots & \ddots & \vdots & \ddots & \vdots \\
S_{n, 1} & \cdots & S_{n, j} & \cdots & S_{n, n}
\end{array}\right] .
$$

The identification equation for the local damage of all the substructures with the global substructural sensitivity matrix can be represented as

$$
S \alpha+o\left(\alpha^{2}\right)=\ddot{X}^{c s}-\ddot{X}^{m s},
$$

where

$$
\begin{aligned}
\alpha & =\left[\begin{array}{lllll}
\alpha_{1}^{T} & \cdots & \alpha_{i}^{T} & \cdots & \alpha_{n}^{T}
\end{array}\right], \\
\ddot{X}^{c s}-\ddot{X}^{m s} & =\left[\begin{array}{lllll}
X_{1}^{T} & \cdots & X_{i}^{T} & \cdots & X_{n}^{T}
\end{array}\right] .
\end{aligned}
$$

The measured acceleration response $\ddot{X}^{m s}$, the corresponding calculated acceleration response $\ddot{X}^{c s}$, and the global substructural sensitivity matrix $S$ in (6) are known while the higher order term $o\left(\alpha^{2}\right)$ can be omitted. The unknown damage index vector $\alpha$ can be determined from (6) with an iterative approach based on Gauss elimination method. The substructural sensitivity matrix $S_{i, j}$ and the difference values of the response vector $X_{i}$ are the basic computing unit in the process of solving. The fractional change increment of the stiffness vector $\Delta \alpha_{i}$ is the result of each iteration, and the final damage index vector $\alpha_{i}$ is obtained by summarizing all the iterative results.

The substructural stiffness matrix is only related to its own damage parameter, so (4) can be also written as

$$
M_{i} \frac{\partial \ddot{x}_{i}}{\partial \alpha_{j}}+C_{i} \frac{\partial \dot{x}_{i}}{\partial \alpha_{j}}+K_{i} \frac{\partial x_{i}}{\partial \alpha_{j}}= \begin{cases}-\frac{\partial K_{i}}{\partial \alpha_{j}} x_{i}-a_{1} \frac{\partial K_{i}}{\partial \alpha_{j}} \dot{x}_{i}+L_{i} \frac{\partial P_{i}}{\partial \alpha_{j}}, & i=j, \\ L_{i} \frac{\partial P_{i}}{\partial \alpha_{j}}, & i \neq j .\end{cases}
$$

In (8), if $i \neq j$, only one term is considered on the righthand side. Obviously, the substructural sensitivity matrix obtained from (8) or $(4), S_{i, j}(i \neq j)$, has less contribution than $S_{i, i}$ to the global substructural sensitivity matrix. A part of the global substructural sensitivity matrix far from the diagonal can be ignored as zero, and then the global 
substructural sensitivity matrix becomes sparse, and the computational processes of Gauss elimination can be simplified.

When the full structure is divided into two substructures, after ignoring low contribution sensitivity matrices, the global substructural sensitivity matrix can be written as

$$
S_{\mathrm{II}}=\left[\begin{array}{cc}
S_{1,1} & 0 \\
0 & S_{2,2}
\end{array}\right]
$$

$$
\begin{aligned}
S_{\mathrm{III}} & =\left[\begin{array}{ccc}
S_{1,1} & S_{1,2} & 0 \\
S_{2,1} & S_{2,2} & S_{2,3} \\
0 & S_{3,2} & S_{3,3}
\end{array}\right] \\
\Delta \alpha_{1}^{k} & =\left(S_{2,1}^{+}+S_{1,1}^{+} S_{1,2} S_{3,2}^{+} S_{3,3} S_{2,3}^{+}-S_{1,1}^{+} S_{1,2} S_{2,2}^{+}\right)\left[\left(S_{2,3} S_{3,3}^{+} S_{3,2} S_{1,2}^{+}-S_{2,2} S_{1,2}^{+}\right) X_{1}+X_{2}-S_{2,3} S_{3,3}^{+} X_{3}\right] \\
\Delta \alpha_{2}^{k} & =S_{1,2}^{+} X_{1}-S_{1,2}^{+} S_{1,1} \Delta \alpha_{1}^{k}, \\
\Delta \alpha_{3}^{k} & =S_{3,3}^{+} X_{3}-S_{3,3}^{+} S_{3,2} \Delta \alpha_{2}^{k} .
\end{aligned}
$$

According to the solution by Gauss elimination method, the $k$ th iterative algorithm can be written as

$$
\begin{aligned}
& \Delta \alpha_{1}^{k}=S_{1,1}^{+} X_{1}, \\
& \Delta \alpha_{2}^{k}=S_{2,2}^{+} X_{2} .
\end{aligned}
$$

Similarly, when the full structure is divided into three substructures, the simplified global substructural sensitivity matrix and the $k$ th iterative algorithm can be written, respectively, as
The substructural method by dividing the full structure into several substructures can effectively reduce structural model, and then the size of response sensitivity matrix is reduced significantly. When there is local damage in different substructures, the following procedure based on the global substructural damage identification method can be performed to assess all the substructures' conditions.

Step 1: Conduct the dynamic measurement in the structure, and assemble the measured responses. Then, set $k=0$.

Step 2: The relationship between the interface forces and the measured responses is constructed, and the interface forces are then identified.

Step 3: Compute the response of the structure $\left(\ddot{X}^{c s}\right)$ and the substructural sensitivity matrices of response $\left(S_{i, j}\right)$ with respect to the local damaged parameters of different substructure.

Step 4: Let $k=k+1$, and identify the $k$ th local change increment of the stiffness with the global substructural sensitivity matrix $S$ in (6).

Step 5: Update the finite element model and repeat Steps 2 to 4 until convergence in (13) is met. The final damage index vector $\alpha$ which is used to represent the change of stiffness can be obtained as $\alpha^{k+1}=\sum_{k+1} \Delta \alpha^{k+1}$.

$\frac{\left\|\alpha^{k+1}-\alpha^{k}\right\|}{\left\|\alpha^{k+1}\right\|}<\operatorname{Tol1} \begin{cases}\text { Toll}=10^{-5} & \text { for the noise free case } \\ \text { Toll }=10^{-3} & \text { for } 5 \% \text { noise level case. }\end{cases}$

The flowchart of the global substructural method is shown in Figure 1.
2.2. The Improved Global Substructural Damage Identification Algorithm. The application of global substructural method to large-span spatial structure can reduce the structural model and the size of sensitivity matrix through dividing the full structure into several substructures, but all the elements need to be considered repeatedly in each iterative computation. When the structural model is too large and complex, it will consume a lot of computation time for damage identification with the global substructural method due to the huge number of elements. To enhance the computational efficiency of damage identification, an improved global substructural method can be proposed by shrinking the scope of assessment in the iterative process.

In this method the damage index is calculated at first identification round, and then a critical value is defined for determining the damaged location, in which those elements with the local damage extent larger than the defined critical value are set as the probable damaged elements. After filtering the probable damaged elements, these elements are independently identified, while regarding the others as intact. Then, all the elements are reviewed to avoid missing the damaged element. Based on the global substructural damage identification method, the improved global substructural damage identification method can be performed as in the following steps:

Step 1: Conduct the dynamic measurement in the structure, and assemble the measured responses. Then, set $k=0$ and $k_{1}=0$.

Step 2: The relationship between the interface forces and the measured responses is constructed, and the interface forces are then identified. Compute the response of the structure and the substructural sensitivity matrices of responses with respect to the local damaged 


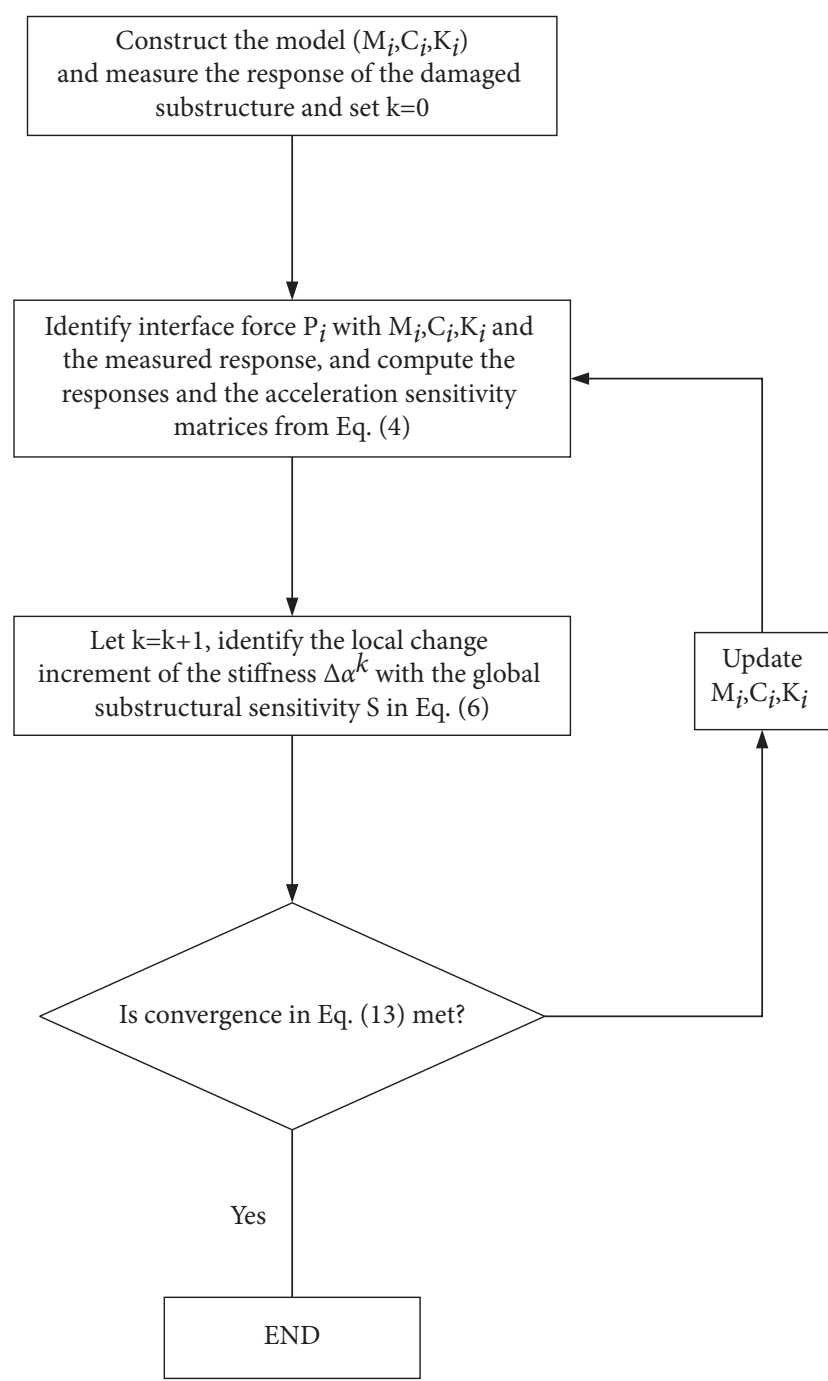

Figure 1: Flowchart of the global substructural method.

parameters of different substructures based on the identified interface forces.

Step 3: Let $k=k+1$, and identify the $k$ th local change increment of the stiffness with the analytical responses and the corresponding substructural sensitivity matrices in Step 2.

Step 4: Update the finite element model and repeat Steps 2 to 3 until convergence in (14) is met. Define a critical value for determining the damaged location. The elements with the obvious stiffness reduction are selected to be updated in the following update procedures (referred to as "damaged elements"), and the stiffness change of the other elements regarded as intact is set to zero. Set $k_{1}=k+1$; the first-round identification is finished.

$\frac{\left\|\alpha^{k+1}-\alpha^{k}\right\|}{\left\|\alpha^{k+1}\right\|}<$ Tol2 $\begin{cases}\text { Tol2 }=10^{-2} & \text { for the noise free case }, \\ \text { Tol2 }=10^{-1} & \text { for } 5 \% \text { noise level case. }\end{cases}$
Step 5: Let $k=k+1$, calculate the responses based on the previous updated model of structure and sensitivities of the "damaged elements" in every substructure, and then identify the $k$ th fractional increment of the damaged parameters of the "damaged elements" $\Delta \alpha_{d}^{k}$.

Step 6: Update the finite element model and repeat Steps 5 until "damaged elements" convergence in (15) is met.

$\frac{\left\|\alpha_{d}{ }^{k+1}-\alpha_{d}{ }^{k}\right\|}{\left\|\alpha_{d}{ }^{k+1}\right\|}<\operatorname{Tol} 3 \begin{cases}\mathrm{Tol} 3=10^{-5} & \text { for the noise free case } \\ \text { Tol3 }=10^{-3} & \text { for } 5 \% \text { noise level case. }\end{cases}$

Step 7: To review the damage identification results, update the finite element model and repeat Steps 2 to 3 until convergence in (14) is met.

The flowchart of the improved global substructural method is shown in Figure 2.

\section{Numerical Simulations}

3.1. Numerical Simulation of Global Substructural Method. The orthogonal spatial square pyramid grid structure as shown in Figure 3 serves for the following study. This structure is modeled using 240 spatial truss finite elements without internal nodes and 72 hinge nodes giving 150 DOFs. The structure is divided into two substructures as shown in Figure 4 . The node number and element number are shown in Figures 3 and 4. Substructure I contains Nodes 1 to 44 and Substructure II contains Nodes 34 to 72 . Nodes 34 to 44 are the interface nodes. The structure is hinge-supported at peripheral nodes, and the location of bearing is shown in Figure 3 as solid blue squares. The upper, lower, and web members are all 3.0 meters long. The cross-sectional area of all members is $0.0028 \mathrm{~m}^{2}$. The plane dimensions of the structure are $18 \mathrm{~m} \times 15 \mathrm{~m}$, and the height of the structure is $2.12 \mathrm{~m}$. The first eight natural frequencies of the structure are $2.724 \mathrm{~Hz}, 4.621 \mathrm{~Hz}, 5.438 \mathrm{~Hz}, 7.029 \mathrm{~Hz}, 7.030 \mathrm{~Hz}, 8.057 \mathrm{~Hz}$, $8.462 \mathrm{~Hz}$, and $8.560 \mathrm{~Hz}$, respectively. Rayleigh damping is adopted for the system, and the two damping coefficients are $a_{1}=0.1077$ and $a_{2}=2.1669 \times 10^{-4}$. The mass density and elastic modulus of material are, respectively, $7.85 \times 10^{3} \mathrm{~kg} /$ $\mathrm{m}^{3}$ and $2.06 \mathrm{GPa}$.

Vertical external loads are applied to the structure at Nodes 26 and 47, and they are, respectively, modeled as

$$
\begin{aligned}
& F_{1}(t)=650 \sin (20 \pi t)+600 \sin (80 \pi t)+550 \sin (160 \pi t), \\
& F_{2}(t)=500 \sin (18 \pi t)+450 \sin (70 \pi t)+450 \sin (210 \pi t),
\end{aligned}
$$

to simulate excitation over a relatively wide range of frequencies.

The sampling rate is $1000 \mathrm{~Hz}$ and the time duration of study is $0.5 \mathrm{~s}$ after the load application. The acceleration responses of the structure are calculated using the Newmark- 


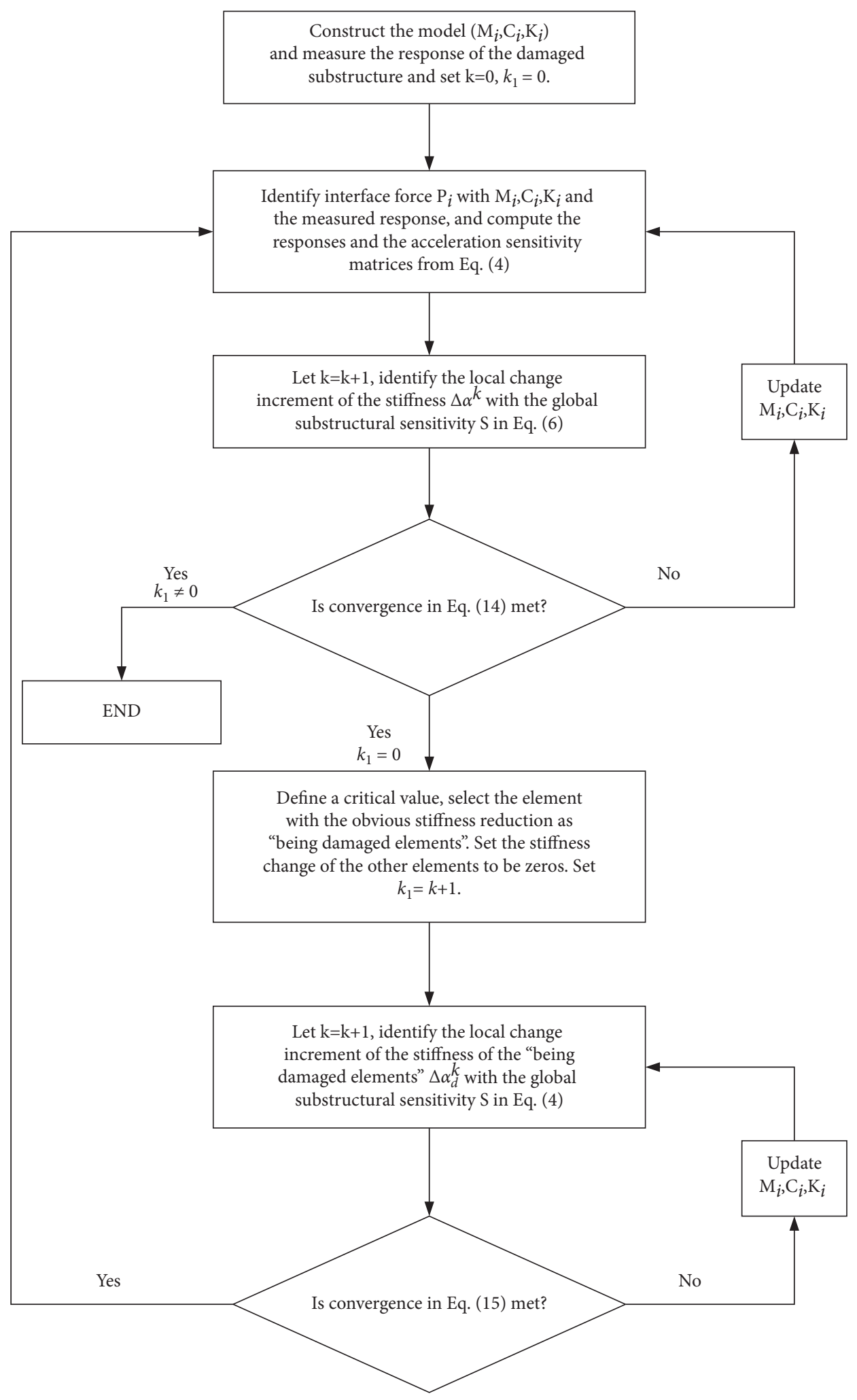

FIGURE 2: Flowchart of the improved global substructural method.

$\beta$ method as the "measured" responses. The sensor location is shown in Figure 3 as red hollow circles.

The initial global substructural sensitivity matrix is obtained with the substructural response sensitivity algorithm. The former 120 columns of matrix are related to the local damaged parameters of Substructure I, and the last 120 columns of matrix are related to the local damaged parameters of Substructure II. To compare the contribution 

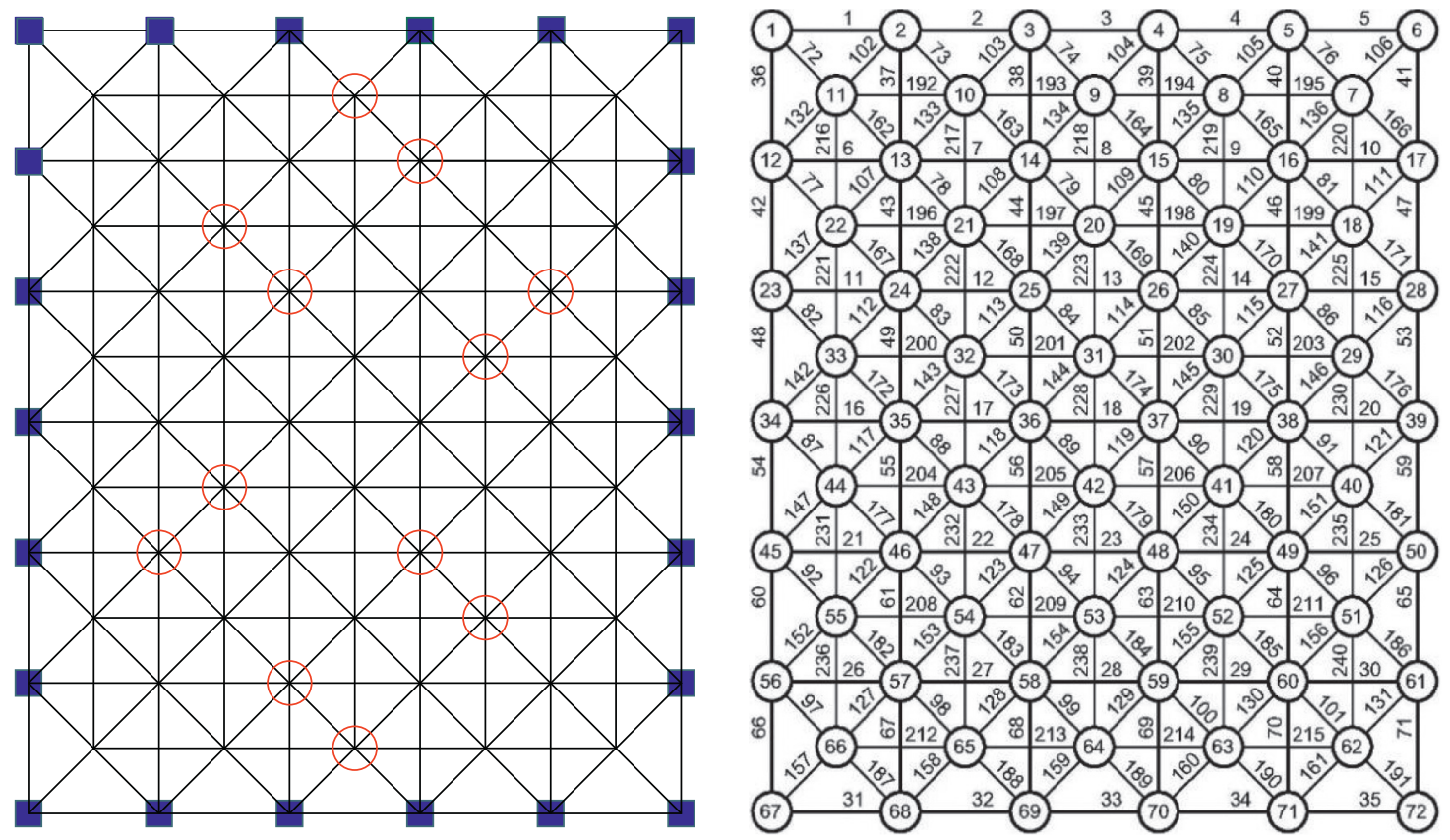

FIgURE 3: An orthogonal spatial square pyramid grid structure and element number system.

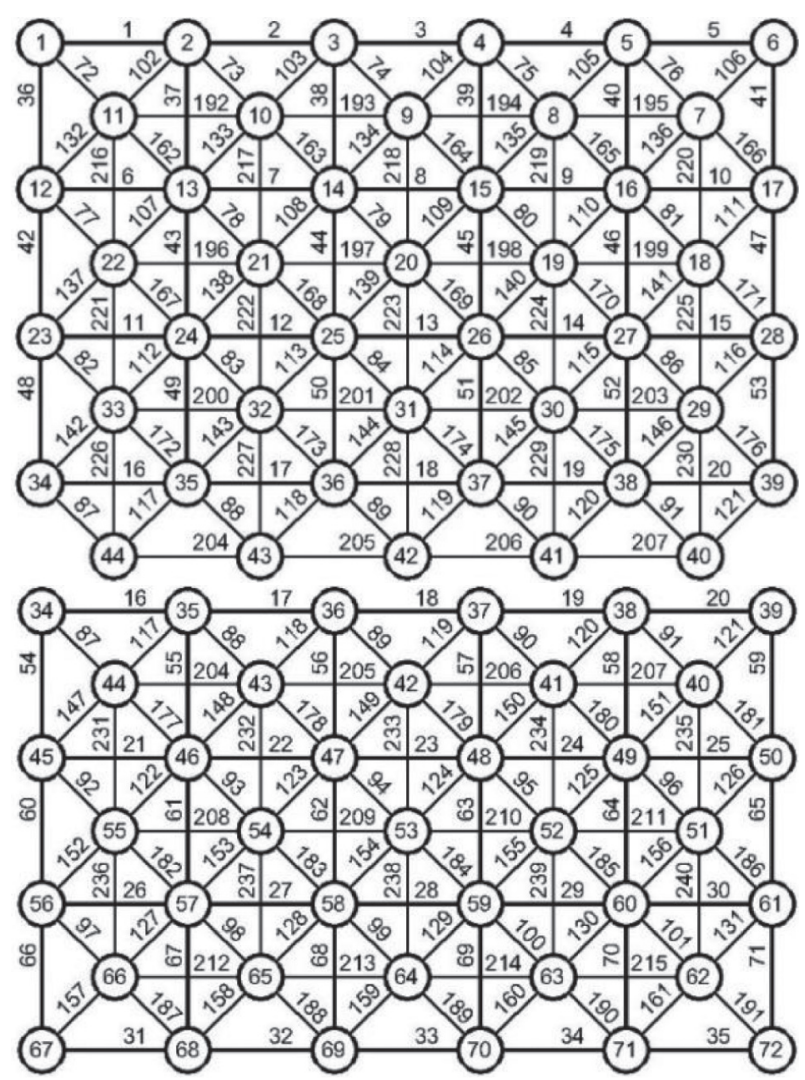

FIGURE 4: Substructure division.

between different substructural sensitivity matrices, every sensitivity vector norm with respect to single local damaged parameter at the different nodes is calculated. The norm of sensitivity vectors at the sensor locations is shown in
Figure 5. The mean of norm of sensitivity vectors is shown in Table 1 . It is noted that the norm of sensitivity vectors with respect to the parameters of Substructure I is greater than that of Substructure II at the nodes belonging to Substructure I, but it is the opposite at the nodes belonging to Substructure II. Accordingly, the substructural sensitivity $S_{i, j}$ $(i \neq j)$ has less contribution than $S_{i, j}(i=j)$ to the global substructural sensitivity matrix.

To evaluate the contribution extent of different substructural sensitivity to the global substructural sensitivity matrix, a ratio is defined as

$$
\text { Ratio }=\frac{\left\|S_{i, j} \Delta \alpha_{j}\right\|}{\left\|\sum_{j} S_{i, j} \Delta \alpha_{j}\right\|} .
$$

Because all the fractional change increments $\Delta \alpha_{i}$ keep the same level under the small local damage, the defined ratio in (17) can represent the influence of one substructural sensitivity in the identification equation. Small stiffness reduction of $0.1 \%$ is simulated in all structural elements, and the initial substructural sensitivity matrices are considered to calculate the ratio. The results of ratio are shown in Table 2, demonstrating that the influence of substructural sensitivity matrix $S_{1,1}$ and $S_{2,2}$ is much larger than the others. In the following numerical simulation, (9) and (10) are applied to assess the condition of structure setting $S_{1,2}$ and $S_{2,1}$ as zero.

Two damage scenarios as shown in Table 3 are studied in this section. $10 \%$ stiffness reduction is assumed as local damage of each selected element in each scenario. The damaged members are located in different substructures.

With the global substructural damage identification method, the damage identification results of the two scenarios are shown in Figure 6. The identified results without noise are consistent with the real damaged scenarios, which 


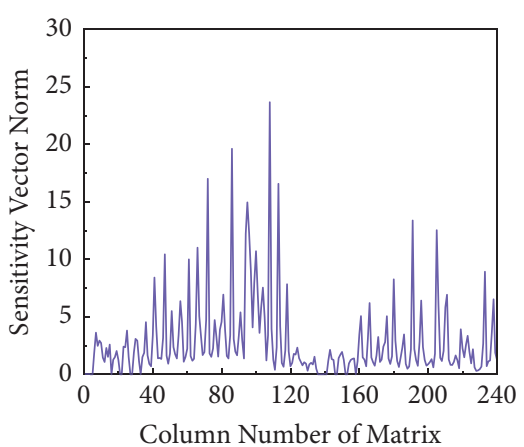

(a)

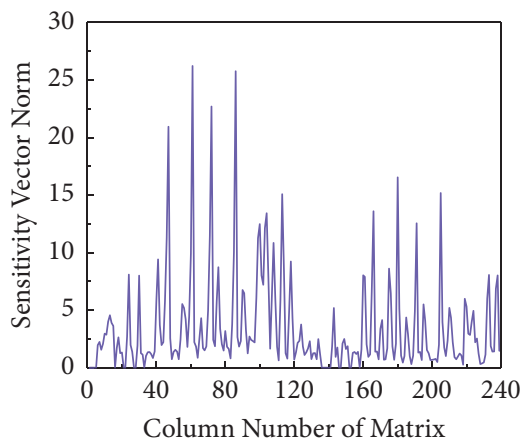

(d)

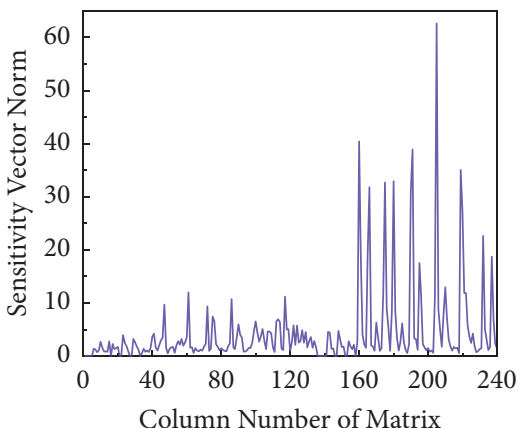

(g)

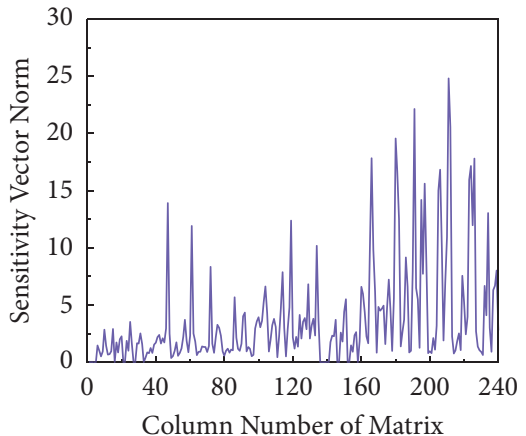

(j)

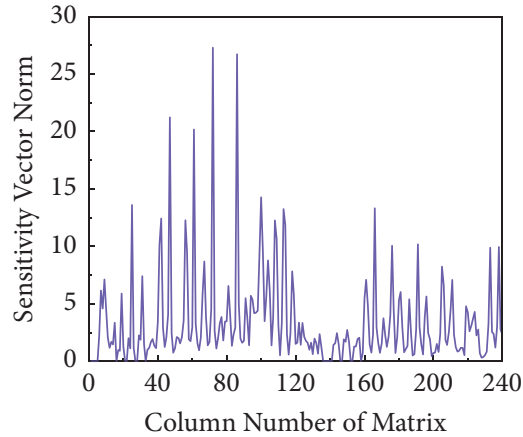

(b)

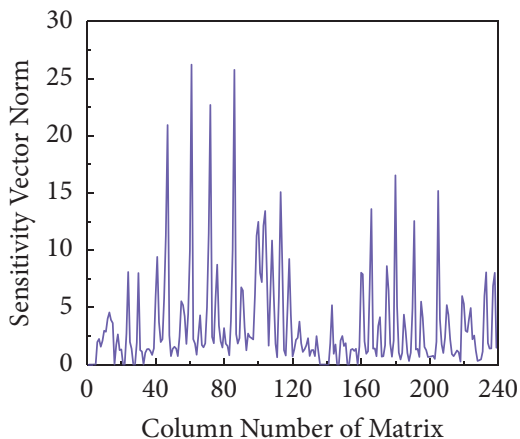

(e)

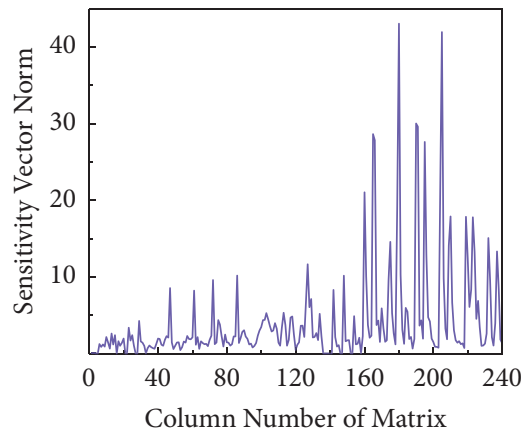

(h)

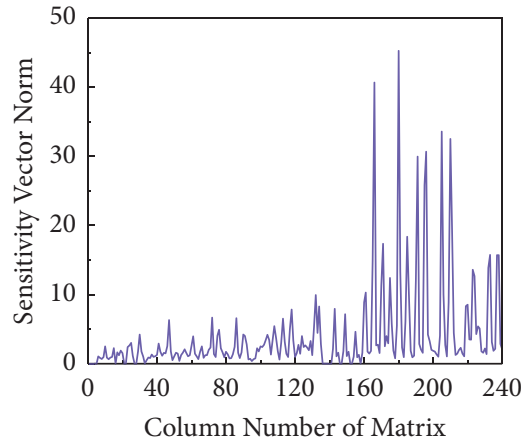

(k)

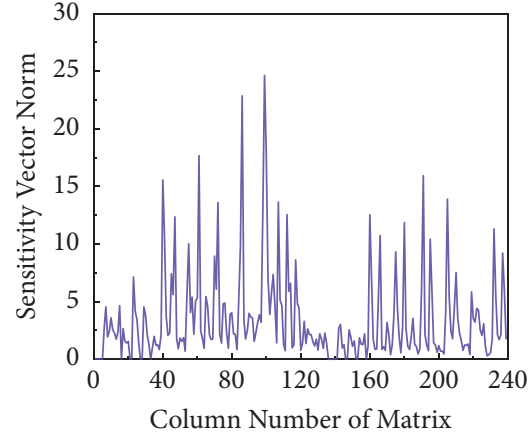

(c)

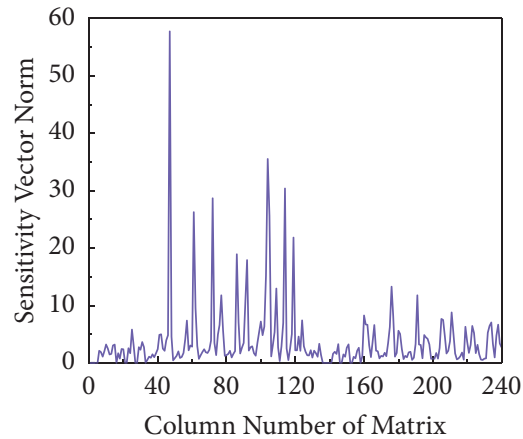

(f)

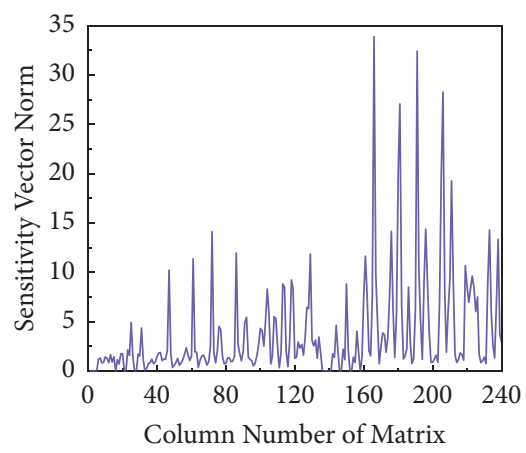

(i)

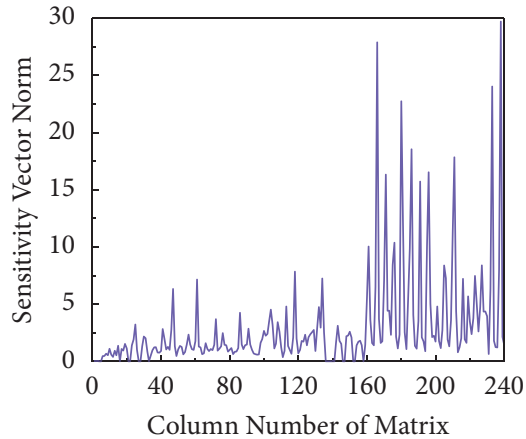

(l)

Figure 5: Sensitivity vector norm. (a) At node 9 belonging to Substructure I. (b) At node 15 belonging to Substructure I. (c) At node 21 belonging to Substructure I. (d) At node 25 belonging to Substructure I. (e) At node 37 belonging to Substructure I. (f) At node 30 belonging to Substructure I. (g) At node 43 belonging to Substructure II. (h) At node 46 belonging to Substructure II. (i) At node 48 belonging to Substructure II. (j) At node 52 belonging to Substructure II. (k) At node 58 belonging to Substructure II. (l) At node 64 belonging to Substructure II. 
TABLE 1: Mean value of sensitivity vector norm.

\begin{tabular}{lcc}
\hline Node number & \multicolumn{2}{c}{ Mean of sensitivity vector norm } \\
& Related to parameters of Sub I & 1.95 \\
9 & 3.68 & 2.44 \\
15 & 4.27 & 2.68 \\
21 & 4.18 & 2.57 \\
25 & 4.32 & 2.11 \\
27 & 4.16 & 2.76 \\
30 & 4.84 & 2.42 \\
All nodes of Sub I & 4.24 & 6.34 \\
43 & 2.45 & 6.24 \\
46 & 2.01 & 5.37 \\
48 & 2.22 & 5.20 \\
52 & 2.11 & 6.09 \\
58 & 1.86 & 4.51 \\
All nodes of Sub II & 1.43 & 5.62 \\
\hline
\end{tabular}

TABLE 2: Contribution extent of substructural sensitivity.

\begin{tabular}{lc}
\hline Index of sensitivity matrix & Ratio \\
\hline$S_{1,1}$ & 0.88 \\
$S_{1,2}$ & 0.33 \\
$S_{2,1}$ & 0.25 \\
$S_{2,2}$ & 0.92 \\
\hline
\end{tabular}

TABle 3: Damage scenarios.

\begin{tabular}{lccc}
\hline Damage scenario & \multicolumn{3}{c}{ Damaged element } \\
& Upper member & Lower member & $\begin{array}{c}\text { Web } \\
\text { member }\end{array}$ \\
\hline Scenario I & 12,46 & 163 & 206,238 \\
Scenario II & 40 & 84,130 & 192,240 \\
\hline
\end{tabular}

indicate that the global substructural damage identification algorithm is correct and accurate. There are some small false positives and negatives in the results, because identification of interface force and simplification of sensitivity matrix will generate errors in iterative process. However, the identification results with $5 \%$ noise in measurements have larger errors than those in the scenario without noise. The mean values and standard deviations of error are shown in Table 4. The identified error of damaged element 46 in Scenario I reaches about $3.8 \%$, but the damage locations can be identified with satisfactory accuracy in both scenarios.

The size of sensitivity matrices is shown in Table 5, and it is noted that the size of sensitivity matrix is reduced significantly in the identification process. This study shows that the global substructural method can be applied for assessing the double-layer lattice space structure with small damage in multiple members distributed in different substructures by incomplete measured acceleration responses $(24 \%$ of responses data are measured in this simulation study). Method. The orthogonal spatial square pyramid grid structure as shown in Figure 7 serves for the following study. This structure is modeled using 800 spatial truss finite elements without internal nodes and 221 hinge nodes giving 543 DOFs. The structure is divided into three substructures as shown in Figure 8, and the node number and element number are shown in Figure 9. Nodes 64 to 84 and Nodes 138 to 158 are the interface nodes. The structure is hinge-supported at peripheral nodes, and the location of bearing is shown in Figure 9 as solid blue squares. The upper, lower, and web members are all 3.0 meters long. The cross-sectional area of all members is $0.0028 \mathrm{~m}^{2}$. The plane dimensions of the structure are $30 \mathrm{~m} \times 30 \mathrm{~m}$, and the height of the structure is 2.12 meters. The first eight natural frequencies of the structure are $0.864 \mathrm{~Hz}, 1.826 \mathrm{~Hz}, 2.564 \mathrm{~Hz}, 3.659 \mathrm{~Hz}, 3.682 \mathrm{~Hz}, 3.985 \mathrm{~Hz}$, $4.144 \mathrm{~Hz}$, and $4.628 \mathrm{~Hz}$, respectively. Rayleigh damping is adopted for the system, and the two damping coefficients are $a_{1}=0.0368$ and $a_{2}=5.9167 \times 10^{-4}$. The mass density and elastic modulus of material are, respectively, $7.85 \times 10^{3} \mathrm{~kg} /$ $\mathrm{m}^{3}$ and $2.06 \mathrm{GPa}$.

Vertical external loads are applied to the structure at Nodes 67, 71, 141, and 145, and they are, respectively, modeled as

$$
\begin{aligned}
& F_{1}(t)=500 \sin (18 \pi t)+450 \sin (70 \pi t)+450 \sin (210 \pi t), \\
& F_{2}(t)=650 \sin (20 \pi t) 600 \sin (80 \pi t)+550 \sin (160 \pi t), \\
& F_{3}(t)=550 \sin (18 \pi t)+500 \sin (50 \pi t)+500 \sin (160 \pi t), \\
& F_{4}(t)=600 \sin (20 \pi t)+550 \sin (30 \pi t)+500 \sin (210 \pi t),
\end{aligned}
$$

to simulate excitation over a relatively wide range of frequencies.

The sampling rate is $1000 \mathrm{~Hz}$ and the time duration of study is $0.5 \mathrm{~s}$ after the load application. The acceleration responses of the structure are calculated using the Newmark$\beta$ method as the "measured" responses. The sensor location is shown in Figure 7 as red hollow circles. Two damaged scenarios as shown in Table 6 are studied in this section. 10\% stiffness reduction is assumed as local damage of selected element in each scenario. Equations (11) and (12) are applied 

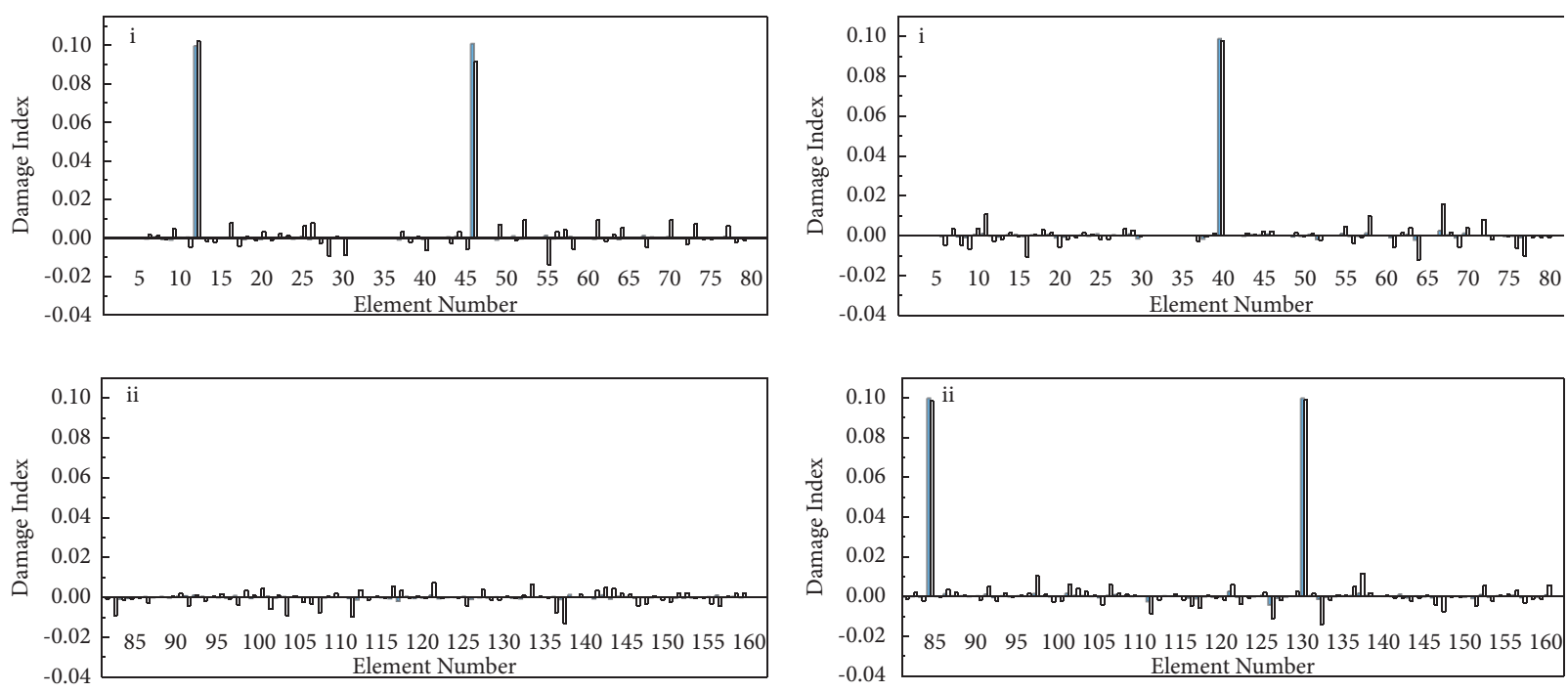

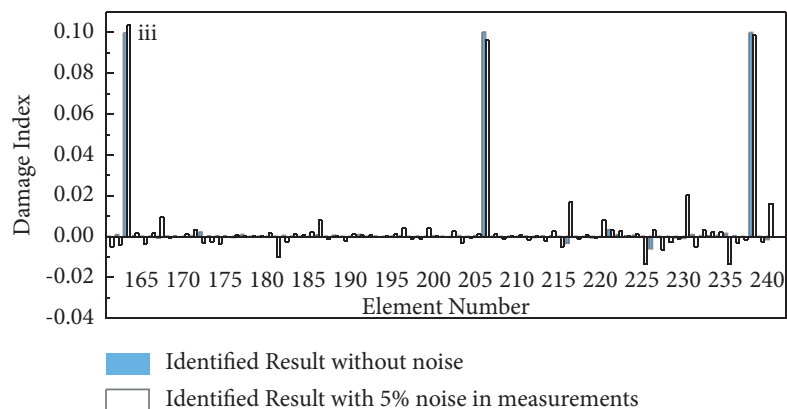

(a)

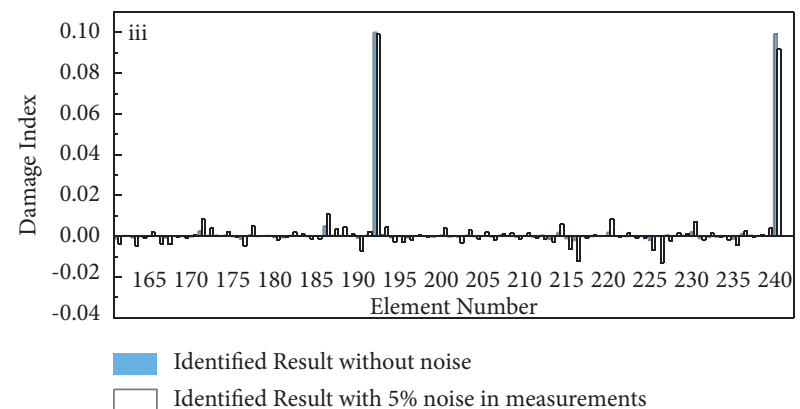

(b)

FIgURE 6: Identified results of global substructural method. (a) Scenario I with (i) part I, (ii) part II, and (iii) part III. (b) Scenario II with (i) part I, (ii) part II, and (iii) part III.

TABLe 4: Calculated error of the identified results.

\begin{tabular}{lcccc}
\hline \multirow{2}{*}{ Damage scenario } & \multicolumn{2}{c}{ With 5\% noise in measurements } & \multicolumn{2}{c}{ Without noise in measurements } \\
& Mean value (\%) & Standard deviation & Mean value (\%) & Standard deviation \\
\hline Scenario I & 1.24 & 1.50 & 0.18 & 0.26 \\
Scenario II & 1.16 & 1.34 & 0.22 & 0.23 \\
\hline
\end{tabular}

TABLE 5: Size of sensitivity matrix.

\begin{tabular}{lc}
\hline Index of sensitivity matrix & Size of sensitivity matrix \\
\hline$S_{\text {II }}$ & $18000 \times 240$ \\
$S_{11}$ & $9000 \times 120$ \\
$S_{22}$ & $9000 \times 120$ \\
\hline
\end{tabular}

to assess the condition of structure setting $S_{1,3}$ and $S_{3,1}$ as zero.

With the improved global substructural method, the damage identification results of the two scenarios without noise in measurements are shown in Figure 8. The identified results are consistent with the real damage scenarios, and there is almost no error, which indicates that the improved global substructural methodology is correct and accurate. The required computation time on a PC with 3.6 GHz Intel Core i7-4790 CPU and $4 \mathrm{~GB}$ memory is shown in Table 7 . Compared with that of the global substructural method, the calculation duration of the improved global substructural method is reduced significantly.

In the first-round identification, the number of elements regarded as "damaged elements" is decided by the defined critical value, being related to the efficiency of next step identification. However, if the defined critical value is too large, some damaged elements may be missing. In order to verify that the improved substructural method is still effective even when there are erroneous judgments in the process of filtering, the critical value is defined as 0.09 in this numerical study. After filtering, the damaged element 500 is to be left out 


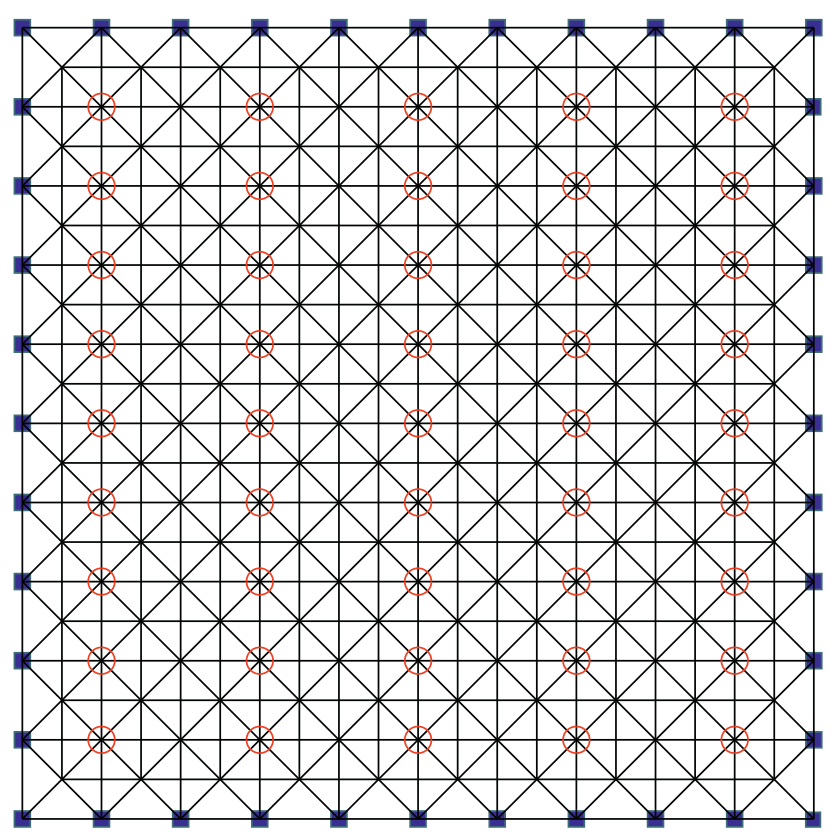

FiguRe 7: An orthogonal spatial square pyramid grid structure.

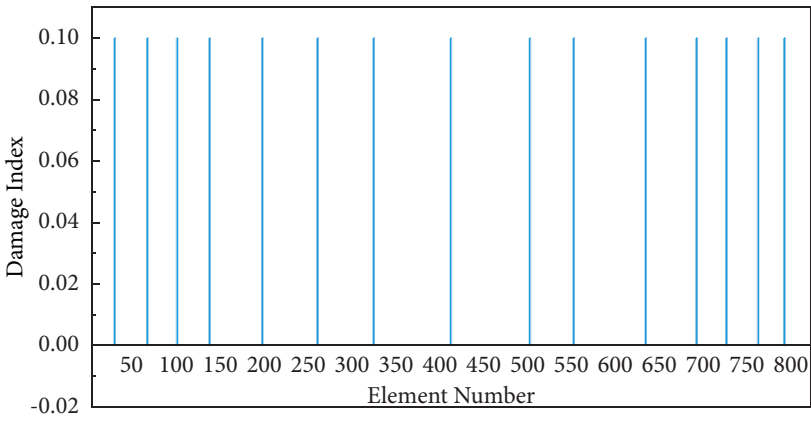

(a)

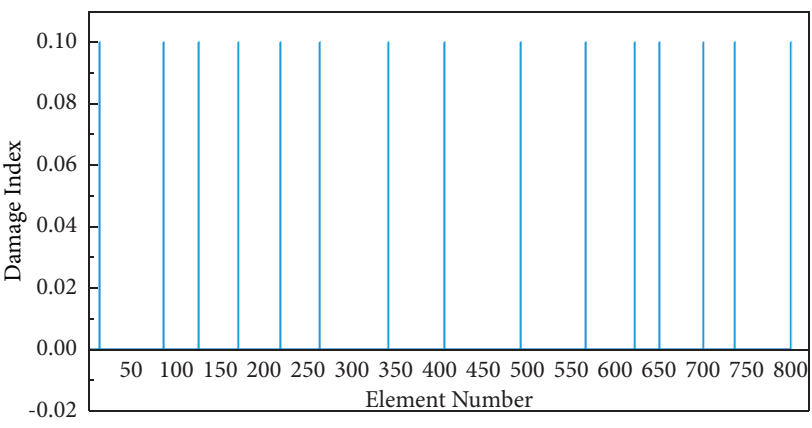

(b)

FIGURE 8: Identified results of improved substructural method. (a) Scenario I. (b) Scenario II.

in Scenario I and the damaged element 736 is to be left out in Scenario II. The identified results of the selected elements of the two scenarios with $5 \%$ noise in measurements are shown in Figure 10, and the locations of erroneous judgment are marked by red circles. The final damage identification results after review of the two scenarios are shown in Figure 11. The correct identified results of damaged location indicate that the accuracy of damage identification is not affected.
The mean value and standard deviation of error are shown in Table 8. It is noted that the error between two methods is extremely close, which indicate that the improved global substructural method is stable for error with $5 \%$ noise while improving the identification speed. $25 \%$ of responses data are obtained for measurement, which is reasonable extent in the practical application. This study shows that the improved global substructural method can be applied for assessing the bigger double-layer lattice space 


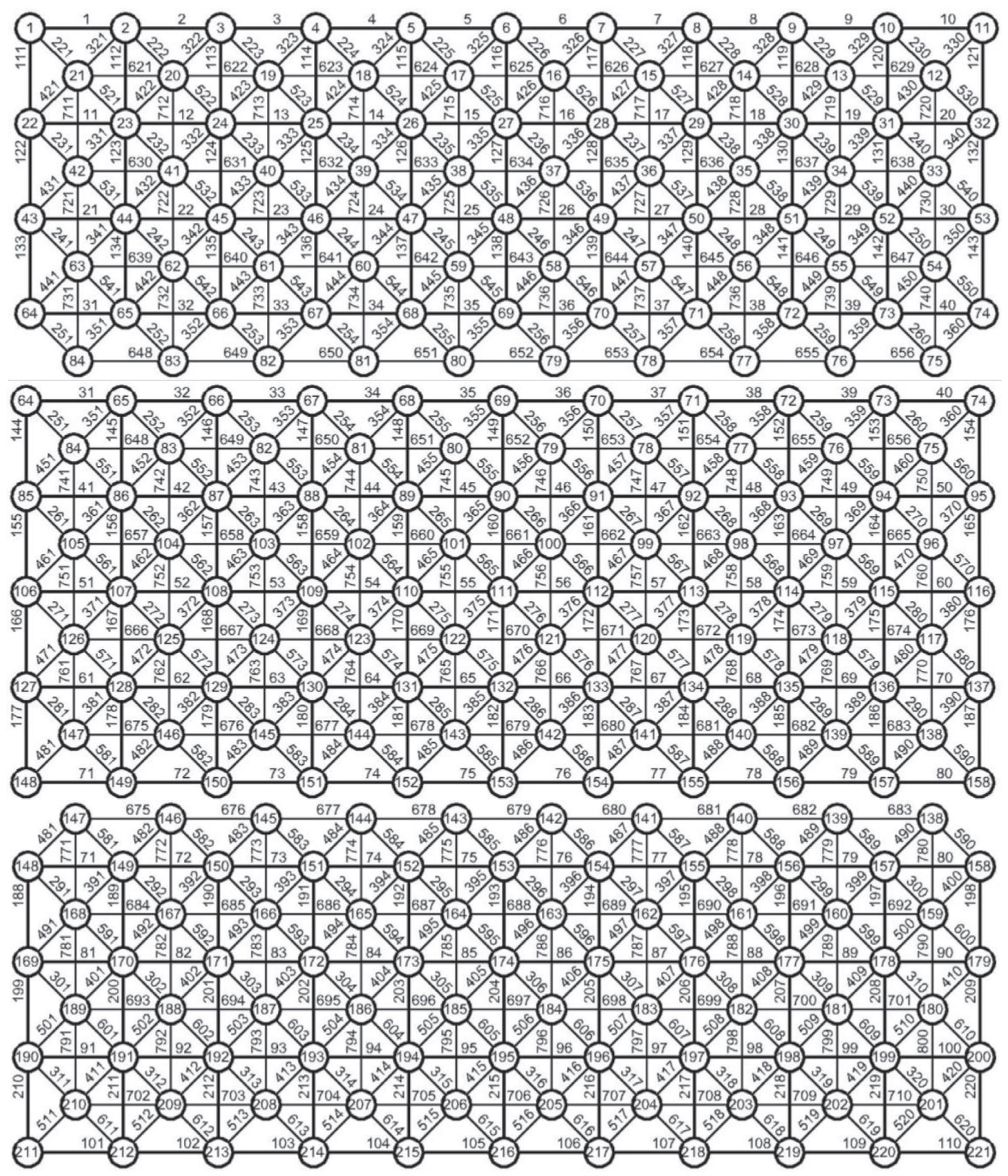

FIgURE 9: Substructure division and element number system.

TABLe 6: Damage scenario.

\begin{tabular}{|c|c|c|c|}
\hline \multirow{2}{*}{ Damage scenario } & \multicolumn{3}{|c|}{ Damaged element } \\
\hline & Upper member & Lower member & Web member \\
\hline Scenario I & $27,64,98,135,195$ & $258,322,410,500,550$ & $632,690,724,760,790$ \\
\hline Scenario II & $12,85,125,170,218$ & $263,341,405,492,566$ & $622,650,700,736,800$ \\
\hline
\end{tabular}

TABle 7: Required computation time (s) for damage identification.

\begin{tabular}{lccccc}
\hline \multirow{2}{*}{ Damage scenario } & \multicolumn{3}{c}{ Improved global substructural method } & \multicolumn{2}{c}{ Global substructural method } \\
& Filtering & Identifying & Review & Total time & \\
\hline Scenario I & 502 & 16 & 407 & 925 & 1108 \\
Scenario II & 526 & 21 & 390 & 937 & 1276 \\
\hline
\end{tabular}




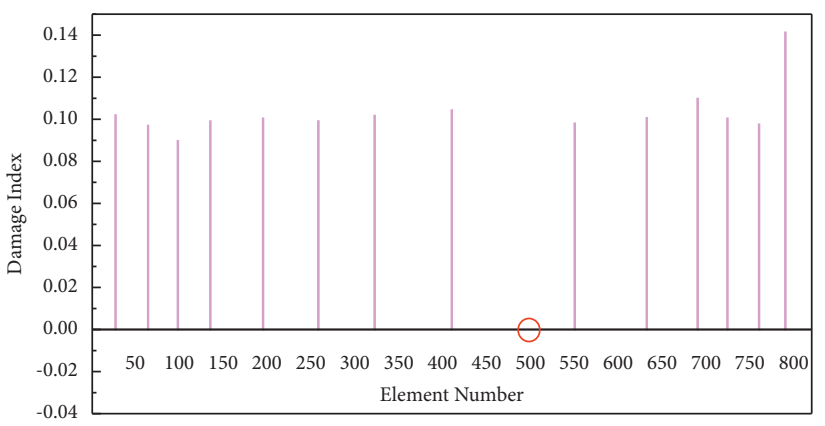

(a)

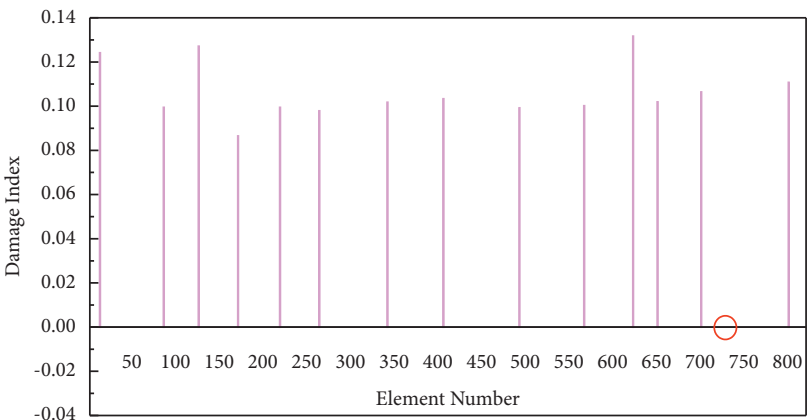

(b)

Figure 10: Identified results of selected elements. (a) Scenario I. (b) Scenario II.

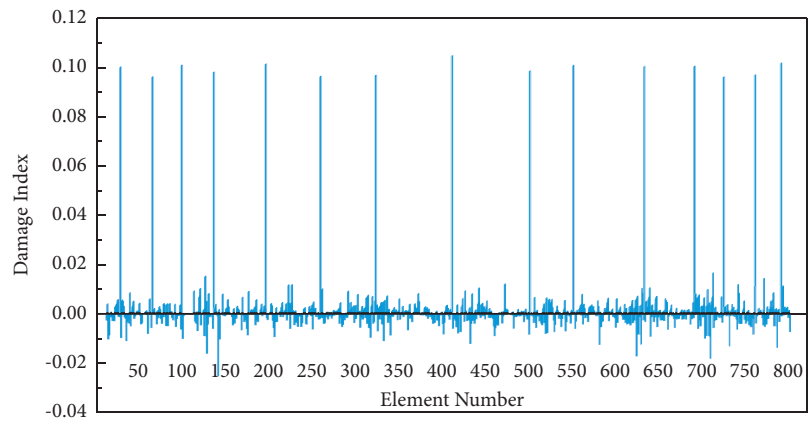

(a)

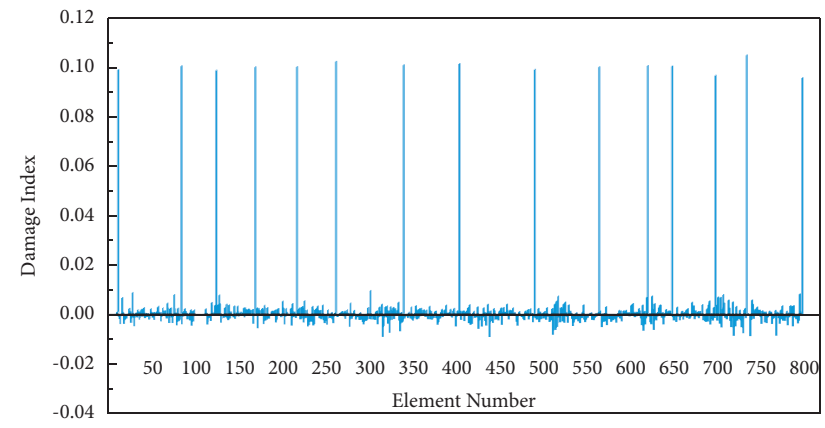

(b)

FIgURE 11: Final identified results after review. (a) Scenario I. (b) Scenario II.

TABLE 8: Calculated error of the identified result with 5\% noise in measurements.

\begin{tabular}{lcccc}
\hline Damage scenario & \multicolumn{2}{c}{$\begin{array}{c}\text { Improved substructural method } \\
\text { Mean value }\end{array}$} & Standard deviation & \multicolumn{2}{c}{$\begin{array}{c}\text { Global substructural method } \\
\text { Mean value }\end{array}$} & 0.71 & Standard deviation \\
\hline Scenario I & 0.61 & 0.67 & 0.50 & 0.79 \\
Scenario II & 0.44 & 0.42 & 0.51 \\
\hline
\end{tabular}

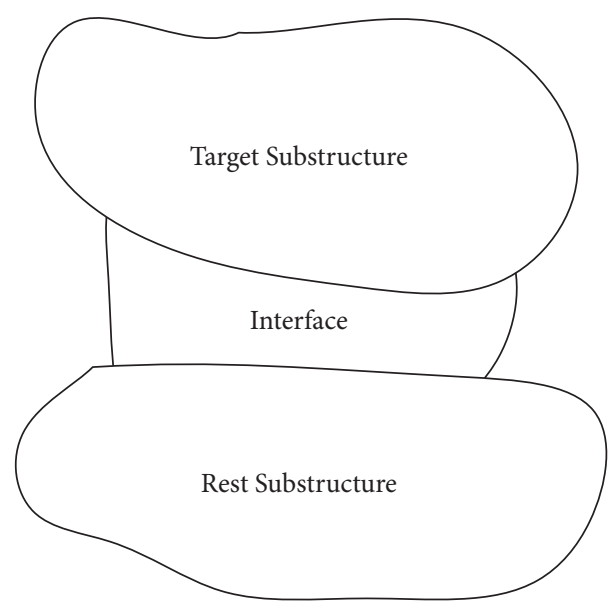

FIGURE 12: Substructure division. 
structure rapidly with dividing substructure to avoid data explosion.

\section{Conclusions and Discussions}

The proposed global substructural damage identification method can assess all the substructures without knowing the conditions of the other parts of the entire structure, so it is a practical method for the health monitoring of large-span spatial structure. The numerical simulation of a double-layer lattice space structure with local damage distributed in different substructures verifies that the new method is effective in identifying the damage of large-span spatial structure by the incomplete dynamic response information. The small damage in multiple members can be accurately assessed without noise, and the damaged location can be correctly identified with $5 \%$ noise in measurements. The divided substructure has less model and smaller sensitivity matrix than the full structure to avoid data explosion, and the appropriate number of sensors ensures the practical feasibility.
The improved global substructural method is proposed to shorten the required computation time, and a larger orthogonal spatial square pyramid grid structure illustrates and verifies this improved global method. The iterative algorithm based on the Gauss elimination of identification is simplified by ignoring a few substructural sensitivities included in the global sensitivity matrix. However, the calculated error will increase with the number of ignored sensitivity matrices growing. The relation between the error and the number of the ignored sensitivity matrices should be further studied. If the number of divided substructures is excessive and the Gauss elimination method suffers difficulty, the Jacobi iteration can be adopted.

\section{Appendix}

\section{A. Explicit Interface Force Identification Method}

The displacement, velocity and acceleration of the substructure at time $t_{i+1}$ can be obtained from Equations (A.1) to (A.3) similar to a full structure [27] as

$$
\begin{aligned}
& \left(x_{\text {sub }}\right)_{i+1}=A_{0} L_{\text {sub }}\left(P_{\text {sub }}\right)_{i+1}+A_{d}\left(x_{\text {sub }}\right)_{i}+A_{v}\left(\dot{x}_{\text {sub }}\right)_{i}+A_{a}\left(\ddot{x}_{\text {sub }}\right)_{i}, \\
& \left(\dot{x}_{\text {sub }}\right)_{i+1}=B_{0} L_{\text {sub }}\left(P_{\text {sub }}\right)_{i+1}+B_{d}\left(x_{\text {sub }}\right)_{i}+B_{v}\left(\dot{x}_{\text {sub }}\right)_{i}+B_{a}\left(\ddot{x}_{\text {sub }}\right)_{i}, \\
& \left(\ddot{x}_{\text {sub }}\right)_{i+1}=C_{0} L_{\text {sub }}\left(P_{\text {sub }}\right)_{i+1}+C_{d}\left(x_{\text {sub }}\right)_{i}+C_{v}\left(\dot{x}_{\text {sub }}\right)_{i}+C_{a}\left(\ddot{x}_{\text {sub }}\right)_{i},
\end{aligned}
$$

where

$$
\begin{aligned}
& \widehat{K}_{\text {sub }}=K_{\text {sub }}+\frac{1}{\beta \Delta t^{2}} M_{\text {sub }}+\frac{\gamma}{\beta \Delta t} C_{\text {sub }} \text {, } \\
& A_{0}=K_{\text {sub }}^{-1} \\
& A_{d}=\widehat{K}_{\text {sub }}^{-1}\left[\frac{1}{\beta \Delta t^{2}} M_{\text {sub }}+\frac{\gamma}{\beta \Delta t} C_{\text {sub }}\right] \\
& A_{v}=\widehat{K}_{\mathrm{sub}}^{-1}\left[\frac{1}{\beta \Delta t} M_{\mathrm{sub}}+\left(\frac{\gamma}{\beta}-1\right) C_{\mathrm{sub}}\right] \\
& A_{a}=\widehat{K}_{\mathrm{sub}}^{-1}\left[\left(\frac{1}{2 \beta}-1\right) M_{\mathrm{sub}}+\frac{\Delta t}{2}\left(\frac{\gamma}{\beta}-2\right) C_{\mathrm{sub}}\right] \\
& B_{0}=\frac{\gamma}{\beta \Delta t} \widehat{K}_{\text {sub }}^{-1} \\
& B_{d}=-\frac{\gamma}{\beta \Delta t} \widehat{K}_{\mathrm{sub}}^{-1} K_{\mathrm{sub}}, \\
& B_{v}=\frac{\gamma}{\beta \Delta t} \widehat{K}_{\text {sub }}^{-1}\left[\left(\frac{\beta \Delta t}{\gamma}-\Delta t\right) K_{\text {sub }}+\frac{1}{\gamma \Delta t} M_{\text {sub }}\right] ; \quad B_{a}=\frac{\gamma}{\beta \Delta t} \widehat{K}_{\text {sub }}^{-1}\left[\left(\frac{\beta \Delta t^{2}}{\gamma}-\frac{\Delta t^{2}}{2}\right) K_{\text {sub }}+\left(\frac{1}{\gamma}-1\right) M_{\text {sub }}\right], \\
& C_{0}=\frac{1}{\beta \Delta t^{2}} \widehat{K}_{\mathrm{sub}}^{-1} \\
& C_{d}=-\frac{1}{\beta \Delta t^{2}} \widehat{K}_{\mathrm{sub}}^{-1} K_{\mathrm{sub}} \\
& C_{v}=\frac{1}{\beta \Delta t^{2}} \widehat{K}_{\mathrm{sub}}^{-1}\left(C_{\mathrm{sub}}+\Delta t K_{\mathrm{sub}}\right) \\
& C_{a}=\frac{1}{\beta \Delta t^{2}} \widehat{K}^{-1}\left[(\gamma-1) \Delta t C_{\text {sub }}-\beta \Delta t^{2}\left(\frac{1}{2 \beta}-1\right) K_{\text {sub }}\right] .
\end{aligned}
$$


Combining (A.1) to (A.3), we have the displacement, velocity, and acceleration at $(i+1)$ th time instant as function of the responses at the $i$ th time instant as

$$
\left[\begin{array}{c}
\left(x_{\text {sub }}\right)_{i+1} \\
\left(\dot{x}_{\text {sub }}\right)_{i+1} \\
\left(\ddot{x}_{\text {sub }}\right)_{i+1}
\end{array}\right]=\left[\begin{array}{c}
A_{0} \\
B_{0} \\
C_{0}
\end{array}\right] L_{\text {sub }}\left(P_{\text {sub }}\right)_{i+1}+\left[\begin{array}{ccc}
A_{d} & A_{v} & A_{a} \\
B_{d} & B_{v} & B_{a} \\
C_{d} & C_{v} & C_{a}
\end{array}\right]\left[\begin{array}{c}
\left(x_{\text {sub }}\right)_{i} \\
\left(\dot{x}_{\text {sub }}\right)_{i} \\
\left(\ddot{x}_{\text {sub }}\right)_{i}
\end{array}\right] .
$$

Equation (A.5) is formulated from the standard Newmark- $\beta$ method for the forward dynamic analysis of a substructure. It can be further rewritten into a general recursive relation as

$$
\begin{gathered}
{\left[\begin{array}{c}
\left(x_{\text {sub }}\right)_{i} \\
\left(\dot{x}_{\text {sub }}\right)_{i} \\
\left(\ddot{x}_{\text {sub }}\right)_{i}
\end{array}\right]=\left[\begin{array}{c}
A_{0} \\
B_{0} \\
C_{0}
\end{array}\right] L_{\text {sub }}\left(P_{\text {sub }}\right)_{i}+\left[\begin{array}{ccc}
A_{d} & A_{v} & A_{a} \\
B_{d} & B_{v} & B_{a} \\
C_{d} & C_{v} & C_{a}
\end{array}\right]\left[\begin{array}{l}
\left(x_{\text {sub }}\right)_{i-1} \\
\left(\dot{x}_{\text {sub }}\right)_{i-1} \\
\left(\ddot{x}_{\text {sub }}\right)_{i-1}
\end{array}\right],} \\
{\left[\begin{array}{c}
\left(x_{\text {sub }}\right)_{i-1} \\
\left(\dot{x}_{\text {sub }}\right)_{i-1} \\
\left(\ddot{x}_{\text {sub }}\right)_{i-1}
\end{array}\right]=\left[\begin{array}{c}
A_{0} \\
B_{0} \\
C_{0}
\end{array}\right] L_{\text {sub }}\left(P_{\text {sub }}\right)_{i-1}+\left[\begin{array}{ccc}
A_{d} & A_{v} & A_{a} \\
B_{d} & B_{v} & B_{a} \\
C_{d} & C_{v} & C_{a}
\end{array}\right]\left[\begin{array}{l}
\left(x_{\text {sub }}\right)_{i-2} \\
\left(\dot{x}_{\text {sub }}\right)_{i-2} \\
\left(\ddot{x}_{\text {sub }}\right)_{i-2}
\end{array}\right],} \\
\vdots \\
{\left[\begin{array}{c}
\left(x_{\text {sub }}\right)_{1} \\
\left(\dot{x}_{\text {sub }}\right)_{1} \\
\left(\ddot{x}_{\text {sub }}\right)_{1}
\end{array}\right]=\left[\begin{array}{c}
A_{0} \\
B_{0} \\
C_{0}
\end{array}\right] L_{\text {sub }}\left(P_{\text {sub }}\right)_{1}+\left[\begin{array}{ccc}
A_{d} & A_{v} & A_{a} \\
B_{d} & B_{v} & B_{a} \\
C_{d} & C_{v} & C_{a}
\end{array}\right]\left[\begin{array}{l}
\left(x_{\text {sub }}\right)_{0} \\
\left(\dot{x}_{\text {sub }}\right)_{0} \\
\left(\ddot{x}_{\text {sub }}\right)_{0}
\end{array}\right],}
\end{gathered}
$$

and the response at time $t_{i}$ can be written as

$$
\left[\begin{array}{c}
\left(x_{\text {sub }}\right)_{i} \\
\left(\dot{x}_{\text {sub }}\right)_{i} \\
\left(\ddot{x}_{\text {sub }}\right)_{i}
\end{array}\right]=\sum_{j=0}^{i-1}\left[\begin{array}{ccc}
A_{d} & A_{v} & A_{a} \\
B_{d} & B_{v} & B_{a} \\
C_{d} & C_{v} & C_{a}
\end{array}\right]^{j}\left[\begin{array}{c}
A_{0} \\
B_{0} \\
C_{0}
\end{array}\right] L_{\text {sub }}\left(P_{\text {sub }}\right)_{i-j}+\left[\begin{array}{ccc}
A_{d} & A_{v} & A_{a} \\
B_{d} & B_{v} & B_{a} \\
C_{d} & C_{v} & C_{a}
\end{array}\right]^{i}\left[\begin{array}{c}
\left(x_{\text {sub }}\right)_{0} \\
\left(\dot{x}_{\text {sub }}\right)_{0} \\
\left(\ddot{x}_{\text {sub }}\right)_{0}
\end{array}\right],
$$

where both indices $i$ and $j$ denote a power of the corresponding matrices.

The measurement matrix $y_{\text {sub }}$ represents the output of the structural system and it can be assembled from the measurements with

$$
y_{\text {sub }}=R_{a} \ddot{x}_{\text {sub }}+R_{v} \dot{x}_{\text {sub }}+R_{d} x_{\text {sub }},
$$

where $R_{a}, R_{v}$ and $R_{d} \in R^{n s \times N}$ are the output influence matrices for the measured acceleration, velocity and displacement respectively, $n s$ is the number of the measured responses and $N$ is the number of DOFs of the selected substructure.

Vector $y_{\text {sub }}$ can be rewritten as

$$
y_{\text {sub }}=\left[\begin{array}{lll}
R_{d} & R_{v} & R_{a}
\end{array}\right]\left\{\begin{array}{c}
x_{\text {sub }} \\
\dot{x}_{\text {sub }} \\
\ddot{x}_{\text {sub }}
\end{array}\right\} .
$$

Let $R=\left[\begin{array}{lll}R_{d} & R_{v} & R_{a}\end{array}\right]$ and substituting Equations (A.7) and (A.9) can be rewritten into the following discrete equation as

$$
y_{\text {sub }}\left(t_{i}\right)=\sum_{j=0}^{i-1} R\left[\begin{array}{ccc}
A_{d} & A_{v} & A_{a} \\
B_{d} & B_{v} & B_{a} \\
C_{d} & C_{v} & C_{a}
\end{array}\right]^{j}\left[\begin{array}{c}
A_{0} \\
B_{0} \\
C_{0}
\end{array}\right] L_{\text {sub }}\left(P_{\text {sub }}\right)_{i-j}+R\left[\begin{array}{ccc}
A_{d} & A_{v} & A_{a} \\
B_{d} & B_{v} & B_{a} \\
C_{d} & C_{v} & C_{a}
\end{array}\right]^{i}\left[\begin{array}{c}
\left(x_{\text {sub }}\right)_{0} \\
\left(\dot{x}_{\text {sub }}\right)_{0} \\
\left(\ddot{x}_{\text {sub }}\right)_{0}
\end{array}\right] .
$$

Assuming zero initial response of the structure and let, 


$$
H_{k}=R\left[\begin{array}{ccc}
A_{d} & A_{v} & A_{a} \\
B_{d} & B_{v} & B_{a} \\
C_{d} & C_{v} & C_{a}
\end{array}\right]^{k}\left[\begin{array}{c}
A_{0} \\
B_{0} \\
C_{0}
\end{array}\right]
$$

Equation (A.10) can be rewritten into a matrix convolution relation from $t_{1}$ to $t_{n t}$ time instants as

$$
Y_{\text {sub }}=\left(H_{\text {sub }}\right)_{L} P_{\text {sub }} \text {, }
$$

where

$$
\begin{aligned}
Y_{\text {sub }} & =\left\{\begin{array}{c}
y_{\text {sub }}\left(t_{1}\right) \\
y_{\text {sub }}\left(t_{2}\right) \\
\vdots \\
y_{\text {sub }}\left(t_{n t}\right)
\end{array}\right\}, \\
\left(H_{\text {sub }}\right)_{L} & =\left[\begin{array}{cccc}
H_{0} L_{\text {sub }} & 0 & \cdots & 0 \\
H_{1} L_{\text {sub }} & H_{0} L_{\text {sub }} & \cdots & 0 \\
\vdots & \vdots & \ddots & \vdots \\
H_{n t-1} L_{\text {sub }} & H_{n t-2} L_{\text {sub }} & \cdots & H_{0} L_{\text {sub }}
\end{array}\right], \\
P_{\text {sub }} & =\left\{\begin{array}{c}
P_{\text {sub }}\left(t_{1}\right) \\
P_{\text {sub }}\left(t_{2}\right) \\
\vdots \\
P_{\text {sub }}\left(t_{n t}\right)
\end{array}\right\} .
\end{aligned}
$$

The inverse problem in Equation (A.12) is ill-posed, and the interface forces are therefore identified through the
Tikhonov regularization technique by minimizing the following objective function.

$$
J\left(P_{\text {sub }}, \lambda\right)=\left\|\left(H_{\text {sub }}\right)_{L} P_{\text {sub }}-Y_{\text {sub }}\right\|^{2}+\lambda\left\|P_{\text {sub }}\right\|^{2},
$$

where $\lambda$ is the regularization parameter obtained using the $L$ curve method.

\section{B.Substructural Damage Identification Method}

The equation of motion of a damped linear structure with multiple DOFs can be written as

$$
M \ddot{x}+C \dot{x}+K x=L P(t),
$$

where $M, C$ and $K$ are the mass, damping and stiffness matrices of the structural system, respectively. $P(t)$ is the vector of external forces on the structure and $L$ is the mapping matrix for the external forces. $\ddot{x}, \dot{x}$ and $x$ are the vectors of acceleration, velocity and displacement responses, respectively. The structure is assumed to exhibit Rayleigh damping for discussion as

$$
C=a_{1} M+a_{2} K,
$$

where $a_{1}$ and $a_{2}$ are the damping coefficients.

A structure can be divided into several substructures and a target substructure can be selected for the assessment. Based on the sub-division of a structure as shown in Figure 12, Equation (B.1) can be rewritten as

$$
\left[\begin{array}{ccc}
M_{r r} & M_{r i} & 0 \\
M_{i r} & M_{i i} & M_{i s} \\
0 & M_{s i} & M_{s s}
\end{array}\right]\left\{\ddot{x}_{r} \ddot{x}_{i} \ddot{x}_{s}\right\}+\left[\begin{array}{ccc}
C_{r r} & C_{r i} & 0 \\
C_{i r} & C_{i i} & C_{i s} \\
0 & C_{s i} & C_{s s}
\end{array}\right]\left\{\begin{array}{c}
\dot{x}_{r} \\
\dot{x}_{i} \\
\dot{x}_{s}
\end{array}\right\}+\left[\begin{array}{ccc}
K_{r r} & K_{r i} & 0 \\
K_{i r} & K_{i i} & K_{i s} \\
0 & K_{s i} & K_{s s}
\end{array}\right]\left\{\begin{array}{c}
x_{r} \\
x_{i} \\
x_{s}
\end{array}\right\}=\left\{\begin{array}{c}
L_{r} P_{r} \\
L_{i} P_{i} \\
L_{s} P_{s}
\end{array}\right\},
$$

where the subscripts $r, i$ and $s$ denote the DOFs of the target substructure, the interface DOFs between the substructures and the DOFs of the rest substructure.
The following equation on DOFs $r$ and $i$ of the structure can be extracted from (B.3) as

$$
\left[\begin{array}{cc}
M_{r r} & M_{r i} \\
M_{i r} & M_{i i}
\end{array}\right]\left\{\ddot{x}_{r} \ddot{x}_{i}\right\}+\left[\begin{array}{cc}
C_{r r} & C_{r i} \\
C_{i r} & C_{i i}
\end{array}\right]\left\{\begin{array}{c}
\dot{x}_{r} \\
\dot{x}_{i}
\end{array}\right\}+\left[\begin{array}{cc}
K_{r r} & K_{r i} \\
K_{i r} & K_{i i}
\end{array}\right]\left\{\begin{array}{c}
x_{r} \\
x_{i}
\end{array}\right\}=\left\{\begin{array}{c}
L_{r} P_{r} \\
L_{i} P_{i}+P_{s}^{\prime}
\end{array}\right\},
$$

where $P_{s}^{\prime}=-M_{i s} \ddot{x}_{s}-C_{i s} \dot{x}_{s}-K_{i s} x_{s}$ is the set of interface forces. The presence of other parts of the structure is represented by the interfacing forces acting on the target substructure. Accurate knowledge of these forces is therefore a requirement for a successful assessment.
The target substructure is selected for study as an individual structure, and the interface forces can be considered as a set of external forces acting on the target substructure. The subscript 'sub' is adopted to represent the substructure. Let, 


$$
\begin{aligned}
& M_{\mathrm{sub}}=\left[\begin{array}{ll}
M_{r r} & M_{r i} \\
M_{i r} & M_{i i}
\end{array}\right], \\
& C_{\text {sub }}=\left[\begin{array}{ll}
C_{r r} & C_{r i} \\
C_{i r} & C_{i i}
\end{array}\right] \text {, } \\
& K_{\text {sub }}=\left[\begin{array}{ll}
K_{r r} & K_{r i} \\
K_{i r} & K_{i i}
\end{array}\right] \text {, } \\
& \ddot{x}_{\text {sub }}=\left\{\ddot{x}_{r} \ddot{x}_{i}\right\} \text {, } \\
& \dot{x}_{\text {sub }}=\left\{\begin{array}{c}
\dot{x}_{r} \\
\dot{x}_{i}
\end{array}\right\} \text {, } \\
& x_{\text {sub }}=\left\{\begin{array}{c}
x_{r} \\
x_{i}
\end{array}\right\} \text {, } \\
& L_{\text {sub }}=\left[\begin{array}{cc}
L_{r} & 0 \\
0 & I
\end{array}\right] \text {, } \\
& P_{\text {sub }}=\left\{\begin{array}{c}
P_{r} \\
P_{s}^{\prime}
\end{array}\right\} \text {, }
\end{aligned}
$$

then the equation of motion of the substructure in Equation (B.4) can be written as

$$
M_{\text {sub }} \ddot{x}_{\text {sub }}+C_{\text {sub }} \dot{x}_{\text {sub }}+K_{\text {sub }} x_{\text {sub }}=L_{\text {sub }} P_{\text {sub }} .
$$

Since matrices $M_{\text {sub }}, C_{\text {sub }}$ and $K_{\text {sub }}$ are positive semidefinite similar to the mass, damping and stiffness matrices of the whole structure, the substructural force identification can also be performed similar to that for a full structure, and the external forces identification method is listed in AppendixA.

Assuming the local damage extent of the $m$ th element in the target substructure as $\alpha_{m}$, the change of the stiffness matrix can be described as

$$
\Delta K_{\text {sub }}=\sum_{m} \alpha_{m}\left(K_{\text {sub }}\right)_{m}
$$

where $\left(K_{\text {sub }}\right)_{m}$ is the stiffness matrix of the $m$ th element in the target substructure. Performing differentiation to both sides of Equation (B.6) with respect to the parameter $\alpha_{m}$, we have

$$
M_{\text {sub }} \frac{\partial \ddot{x}_{\text {sub }}}{\partial \alpha_{m}}+C_{\text {sub }} \frac{\partial \dot{x}_{\text {sub }}}{\partial \alpha_{m}}+K_{\text {sub }} \frac{\partial x_{\text {sub }}}{\partial \alpha_{m}}=-\frac{\partial K_{\text {sub }}}{\partial \alpha_{m}} x_{\text {sub }}-a_{1} \frac{\partial K_{\text {sub }}}{\partial \alpha_{m}} \dot{x}_{\text {sub }}+L_{\text {sub }} \frac{\partial P_{\text {sub }}}{\partial \alpha_{m}}
$$

Equation (B.8) is of the same form as similar equations for the response sensitivity of a full structure [8] except one extra term (the third term) on the right-hand side. When only the first two terms on the right are included, the obtained response sensitivity is for the substructure with an non-varying interface force. Equation (B.8) is solved again by keeping the third term on the right to get the response sensitivity derived from the interface force sensitivity alone. The response sensitivity derived from the interface force sensitivity is noted to be significant [26] and it cannot be ignored in the calculation of the response sensitivity of the substructure.
Vector $y_{\mathrm{sub}}^{\alpha m}$ represents the response sensitivity vector of the substructural system and similar to Equation (A.8) it can be assembled as

$$
y_{\text {sub }}^{\alpha m}=R_{a} \frac{\partial \ddot{x}_{\mathrm{sub}}}{\partial \alpha_{m}}+R_{v} \frac{\partial \dot{x}_{\mathrm{sub}}}{\partial \alpha_{m}}+R_{d} \frac{\partial x_{\mathrm{sub}}}{\partial \alpha_{m}} .
$$

Let,

$$
L_{\text {sub }}^{\alpha m}=\left[\begin{array}{lll}
-\frac{\partial K_{\text {sub }}}{\partial \alpha_{m}}-a_{1} \frac{\partial K_{\text {sub }}}{\partial \alpha_{m}} & 0
\end{array}\right] .
$$

Combining Equations (B.8) and (B.9) and assuming zero initial conditions, similar to Equations (A.1) to (A.10) the following solution of the sensitivity can be obtained.

$$
y_{\text {sub }}^{\alpha m}\left(t_{i}\right)=\sum_{j=0}^{i-1} R\left[\begin{array}{ccc}
A_{d} & A_{v} & A_{a} \\
B_{d} & B_{v} & B_{a} \\
C_{d} & C_{v} & C_{a}
\end{array}\right]^{j}\left[\begin{array}{c}
A_{0} \\
B_{0} \\
C_{0}
\end{array}\right]\left(L_{\text {sub }}^{\alpha m}\left\{\begin{array}{c}
x_{\text {sub }} \\
\dot{x}_{\text {sub }} \\
\ddot{x}_{\text {sub }}
\end{array}\right\}_{i-j}+L_{\text {sub }}\left(\frac{\partial P_{\text {sub }}}{\partial \alpha_{m}}\right)_{i-j}\right),
$$

Based on Equation (A.12), the sensitivity of the interface force can be defined as

$$
\frac{\partial P_{\text {sub }}}{\partial \alpha_{m}}=\left[\left(H_{\text {sub }}\right)_{L}\right]^{-1} \frac{\partial\left(H_{\text {sub }}\right)_{L}}{\partial \alpha_{m}}\left[\left(H_{\text {sub }}\right)_{L}\right]^{-1} Y_{\text {sub }}
$$

and identified based on the explicit Newmark- $\beta$ method [27],

$$
\left\{\begin{array}{c}
x_{\text {sub }} \\
\dot{x}_{\text {sub }} \\
\ddot{x}_{\text {sub }}
\end{array}\right\}=\sum_{j=0}^{i-1}\left[\begin{array}{ccc}
A_{d} & A_{v} & A_{a} \\
B_{d} & B_{v} & B_{a} \\
C_{d} & C_{v} & C_{a}
\end{array}\right]^{j}\left[\begin{array}{c}
A_{0} \\
B_{0} \\
C_{0}
\end{array}\right] L_{\text {sub }}\left(P_{\text {sub }}\right)_{i-j \cdot} .
$$

Similar to Equations (A.12) and (A.13), let 


$$
\begin{aligned}
H_{k}^{w}= & {\left[\begin{array}{ccc}
A_{d} & A_{v} & A_{a} \\
B_{d} & B_{v} & B_{a} \\
C_{d} & C_{v} & C_{a}
\end{array}\right]^{k}\left[\begin{array}{c}
A_{0} \\
B_{0} \\
C_{0}
\end{array}\right], } \\
\left(H_{\text {sub }}^{w}\right)_{L}= & {\left[\begin{array}{cccc}
H_{0}^{w} L_{\text {sub }} & 0 & \cdots & 0 \\
H_{1}^{w} L_{\text {sub }} & H_{0}^{w} L_{\text {sub }} & \cdots & 0 \\
\vdots & \vdots & \ddots & \vdots \\
H_{n t-1}^{w} L_{\text {sub }} & H_{n t-2}^{w} L_{\text {sub }} & \cdots & H_{0}^{w} L_{\text {sub }}
\end{array}\right], } \\
\left(H_{\text {sub }}^{\alpha m}\right)_{L}= & {\left[\begin{array}{cccc}
H_{0} L_{\text {sub }}^{\alpha m} & 0 & \cdots & 0 \\
H_{1} L_{\text {sub }}^{\alpha m} & H_{0} L_{\text {sub }}^{\alpha m} & \cdots & 0 \\
\vdots & \vdots & \ddots & \vdots \\
H_{n t-1} L_{\text {sub }}^{\alpha m} & H_{n t-2} L_{\text {sub }}^{\alpha m} & \cdots & H_{0} L_{\text {sub }}^{\alpha m}
\end{array}\right] . }
\end{aligned}
$$

The response sensitivity considering the interface force effect can be obtained as,

$$
Y_{\text {sub }}^{\alpha m}=\left(\left(H_{\text {sub }}^{\alpha m}\right)_{L}\left(H_{\text {sub }}^{w}\right)_{L}+\frac{\partial\left(H_{\text {sub }}\right)_{L}}{\partial \alpha_{m}}\right)\left[\left(H_{\text {sub }}\right)_{L}\right]^{-1} Y_{\text {sub }} \text {, }
$$

and the sensitivity matrix $\mathbf{S}_{\text {sub }}$ for the substructure with $N E$ elements can be defined as

$$
S_{\mathrm{sub}}=\left[\begin{array}{llll}
Y_{\mathrm{sub}}^{\alpha 1} & Y_{\mathrm{sub}}^{\alpha 2} & \cdots & Y_{\mathrm{sub}}^{\alpha N E}
\end{array}\right]
$$

\section{Data Availability}

Any reader interested in the data can contact the corresponding author through e-mail.

\section{Conflicts of Interest}

The authors declare no conflicts of interest.

\section{Acknowledgments}

This study was jointly sponsored by National Key R\&D Programs of China (Grant nos. 2017YFC1500601 and 2017YFC1500602); Scientific Research Fund of Institute of Engineering Mechanics, China Earthquake Administration (Grant Nos. 2021B01 and 2021EEEVL0308); and Program for Innovative Research Team in China Earthquake Administration.

\section{References}

[1] G. B. Nie, J. W. Dai, C. X. Zhang, and X. D. Zhi, "Failure patterns of large span space structures in Lushan earthquake and numerical simulation," China Civil Engineering Journal, vol. 48, no. 4, pp. 1-6, 2015.

[2] O. S. Salawu, "Detection of structural damage through changes in frequency: a review," Engineering Structures, vol. 19, no. 9, pp. 718-723, 1997.

[3] O. Vera and N. Chouw, "Damage identification of unreinforced masonry panels using vibration-based techniques," Shock and Vibration, vol. 2017, Article ID 9161025, 14 pages, 2017.
[4] S. W. Doebling, C. R. Farrar, and M. B. Prime, "A summary review of vibration-based damage identification methods," The Shock and Vibration Digest, vol. 30, no. 2, pp. 91-105, 1998.

[5] X. Liu, N. A. J. Lieven, and P. J. Escamilla-Ambrosio, "Frequency response function shape-based methods for structural damage localization," Mechanical Systems and Signal Processing, vol. 23, no. 2009, pp. 1243-1259, 2009.

[6] O. Huth, G. Feltrin, J. Maeck, and K. Nedim, "Damage identification using modal data: experiences on a prestressed concrete bridge," Journal of Structural Engineering, vol. 131, no. 12, pp. 1898-1910, 2005.

[7] X. Wang, K. Liu, H. Liu, and Y. He, "Damping identificationwith acceleration measurements based on sensitivity en-hancement method," Shock and Vibration, vol. 2018, Article ID 6476783, 16 pages, 2018.

[8] K. Liu, S. S. Law, Y. Xia, and X. Q. Zhu, "Singular spectrum analysis for enhancing the sensitivity in structural damage detection," Journal of Sound and Vibration, vol. 333, no. 2014, pp. 392-417, 2014.

[9] W. Ji, Y. Song, and B. Liang, "Damage diagnosis in truss by modal analysis," Journal of Shenyang Jianzhu University (Natural Science), vol. 26, no. 2, pp. 232-237, 2010.

[10] W. Ji, Y. Song, B. Liang, and Y. Song, "Numerical simulation research of for damage diagnosis of truss structure by ANSYS," Journal of Wuhan University of Technology, vol. 30, no. 2, pp. 285-288, 2006.

[11] Y. Song, L. Zhang, and Z. Liu, "Damage identification of space truss with incomplete measured data," China Civil Engineering Journal, vol. 42, no. 1, pp. 10-14, 2009.

[12] W. Gao, W. Liu, and C. Qian, "Damage detection of space truss using residual modal force and modal strain energy," Engineering Mechanics, vol. 24, no. 5, pp. 93-100, 2007.

[13] J. Wu and Y. Zhang, "Damage identification of grid structures based on BP neural networks and experimental study," World Earthquake Engineering, vol. 21, no. 4, pp. 71-76, 2005.

[14] C. Liu, Y. Zhang, and J. Wu, "Substructure-oriented damage localization method of the spatial lattice structure," Building Structure, vol. 44, no. 6, pp. 79-84, 2014.

[15] H. He and W. Yan, "Structural damage detection with wavelet support vector machine:Introduction and applications," Structural Control and Health Monitoring, vol. 14, no. 1, pp. 162-176, 2007.

[16] J. Teng and W. Lu, "Structural damage identification method based on multi-type sensors," Journal of Southeast University (Natural Science Edition), vol. 40, no. 3, pp. 538-542, 2010.

[17] J. Teng and W. Lu, "Damage identification method based on structural dynamic characteristics and strain measurements," Health Monitoring of Structural and Biological Systems, vol. 729520, no. 9, 2009.

[18] J. Teng and S. Yao, "A method of earthquake-induced latticed shell damage detection by wavelet packet analysis," Engineering Mechanics, vol. 28, no. 9, pp. 183-188, 2011.

[19] K. Yuen, "Substructure identification and health monitoring using noisy response measurements only," Computer-Aided Civil and Infrastructure Engineering, vol. 21, no. 4, pp. 280291, 2006.

[20] K. Yuen and K. Huang, "Real-time substructural identification by boundary force modelling," Structural Control and Health Monitoring, vol. 25, no. 4, pp. 1-18, 2018.

[21] K. Yuen and K. Huang, "Identifiability-enhanced Bayesian frequency-domain substructure identification," ComputerAided Civil and Infrastructure Engineering, vol. 33, no. 9, pp. 800-812, 2018. 
[22] H. A. Jahn, "Improvement of an approximate set of latent roots and modal columns of a matrix by methods akin to those of classical perturbation theory," Quarterly Journal of Mechanics \& Applied Mathematics, vol. 1, no. 1, pp. 132-144, 1948.

[23] W. Deng, J. Xu, H. Zhao, and Y. Song, "A novel gate resource allocation method using improved PSO-based QEA," IEEE Transactions on Intelligent Transportation Systems, vol. 2020, pp. 1-9, 2020.

[24] W. Deng, S. Shang, X. Cai et al., "Quantum differential evolution with cooperative coevolution framework and hybrid mutation strategy for large scale optimization," Knowledge-Based Systems, vol. 224, no. 2021, pp. 1-14, Article ID 107080, 2021.

[25] W. Deng, J. Xu, X.-Z. Gao, and H. Zhao, "An enhanced MSIQDE algorithm with novel multiple strategies for global optimization problems," IEEE Transactions on Systems, Man, and Cybernetics: Systems, vol. 2020, pp. 1-10, 2020.

[26] K. Liu, S. S. Law, and X. Q. Zhu, "Substructural condition assessment based on force identification and interface force sensitivity," International Journal of Structural Stability and Dynamics, vol. 15, no. 2, Article ID 1450046, 2015.

[27] K. Liu, S. S. Law, X. Q. Zhu, and Y. Xia, "Explicit form of an implicit method for inverse force identification," Journal of Sound and Vibration, vol. 333, no. 3, pp. 730-744, 2014. 